NASA Technical Memorandum 105955

ICOMP-92-25; CMOTT-92-13

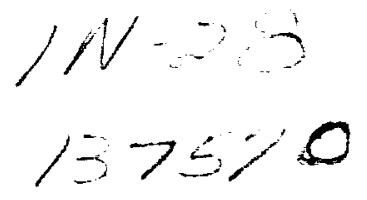

$p-70$

\title{
Experiments on a Round Turbulent Buoyant Plume
}

\author{
Aamir Shabbir \\ Institute of Computational Mechanics in Propulsion \\ Lewis Research Center \\ Cleveland, Ohio \\ and \\ William K. George \\ State University of New York at Buffalo \\ Buffalo, New York
}


$=$ 


\title{
Experiments on a Round Turbulent Buoyant Plume
}

\author{
Aamir Shabbir \\ Center for Modeling of Turbulence and Transition \\ Institute of Computational Mechanics in Propulsion \\ Lewis Research Center \\ Cleveland, OH 44135 \\ and \\ William K. George \\ State University of New York at Buffalo \\ Buffalo, NY 14260
}

\begin{abstract}
This paper reports a comprehensive set of hot-wire measurements of a round buoyant plume which was generated by forcing a jet of hot air vertically up into quiescent environment. The boundary conditions of the experiment were measured, and are documented in the present paper in an attempt to sort out the contradictory mean flow results from the earlier studies. The ambient temperature was monitored to insure that the facility was not stratified and that the experiment was conducted in a neutral environment. The axisymmetry of the flow was checked by using a planer array of sixteen thermocouples and the mean temperature measurements from these are used to supplement the hot-wire measurements. The source flow conditions were measured so as to ascertain the rate at which the buoyancy was added to the flow. The measurements conserve buoyancy within $10 \%$. The results are used to carry out the balances of the mean energy and momentum differential equations. In the mean energy equation it is found that the vertical advection of the energy is primarily balanced by the radial turbulent transport. In the mean momentum equation the vertical advection of momentum and the buoyancy force balance the radial turbulent transport. The buoyancy force is the second largest term in this balance and is responsible for the wider (and higher) velocity profiles in plumes as compared to jets. Budgets of the temperature variance and turbulence kinetic energy are also carried out in which thermal and mechanical dissipation rates are obtained as the closing terms. Similarities and differences between the two balances are discussed. It is found that even though the direct affect of buoyancy on turbulence, as evidenced by the buoyancy production term, is substantial, most of the turbulence is produced by shear. This is in contrast to the mean velocity field where the affect of buoyancy force is quite strong. Therefore, it is concluded that in a buoyant plume the primary affect of buoyancy on turbulence is indirect, and enters through the mean velocity field (giving larger shear production).
\end{abstract}




\section{Preliminaries}

\subsection{Introduction}

Buoyancy plays an important role in a number of fluid flows of environmental and technological importance. Some examples are: vertical motion of air in the atmosphere; spreading of the smoke and other pollutants in the atmosphere; dispersal of volcano exhaust and water outfalls; and numerous industrial problems involving the natural convection cooling of isolated sources. The buoyancy force in such flows can either be caused by a heat source, as in a smoke stack, or it can be caused by the introduction of one fluid into another fluid of different density, as is in a water outfall. Once the fluid is set into motion, its velocity field affects the thermal field, and vice-versa. The initial state of laminar motion very quickly changes into turbulence and the flow starts to spread radially by entraining ambient fluid into the main flow. The laboratory plume is an idealization of these naturally occurring flows and it allows us to study the influence of buoyancy on turbulence. It can be shown that regardless of the kind of source, all plumes are dynamically similar as long as the density differences between the flow and the ambient are small as compared to some reference density in the flow. Therefore, for convenience, and with no loss of generality, the source of buoyancy in this paper will be assumed to be heat and the density differences will be characterized by the temperature differences.

In the analysis of such flows the ambient fluid is assumed to be at rest. If it's temperature does not change with height, the environment is termed as neutral. If the ambient temperature increases with height the environment is called unstable, and provides an additional source of buoyancy force. In a stable environment the temperature decreases with height and the growth of the plume is suppressed. This paper reports the hot-wire measurements of a round turbulent buoyant plume in a neutral environment. 


\subsection{Historical Development}

The plume has been the subject of study since the similarity analysis of Zel'dovich (1937). Schmidt (1941) used mixing length type hypotheses to obtain expressions for the mean velocity and temperature profiles for both the plane and the axisymmetric geometries and compared the results with his own measurements. Batchelor (1954) proposed similarity solutions for turbulent plumes for both the plane and the round geometries in neutral and stratified environments. For the round plume in a neutral environment, the mean vertical velocity and buoyancy field were shown to be given by

$$
\begin{aligned}
W & =F_{o}^{1 / 3} z^{-1 / 3} f(r / z) \\
g \frac{\Delta \rho}{\rho_{\infty}} & =F_{o}^{2 / 3} z^{-5 / 3} t(r / z)
\end{aligned}
$$

where $F_{o}$ is the rate at which buoyancy is added at the source, $z$ is the height, $g$ is the gravitational acceleration, $\rho_{\infty}$ is the reference density (usually at infinity) and $\Delta \rho$ is the difference between the reference and the local mean density. The similarity variable is $\eta=r / z$ and the functions $f$ and $t$ are to be determined from experiments. It is common to represent these by Gaussian functions of the form

$$
\begin{gathered}
f(\eta)=A_{u} e^{\left(-B_{\imath} \eta^{2}\right)} \\
t(\eta)=A_{t} e^{\left(-B_{t} \eta^{2}\right)}
\end{gathered}
$$

Rouse et al. (1952) also obtained the similarity relations for the plane and axisymmetric plumes and experimentally verified their functional forms. They generated plumes by using gas burners, arranged in the appropriate formation, and then used thermocouples and wind-vane anemometers to measure the mean buoyancy and mean vertical velocity .

Morton et al. (1956) extended the analysis to non-similar situations by assuming that the entrainment rate was proportional to some local characteristic velocity. By calculating 
the entrainment constant from the experiments, they made predictions of the heights to which plumes can rise under different ambient conditions.

Morton (1959) examined how a source of momentum and buoyancy evolves into a plume. The distance over which this evolution occurs can be characterized by the length scale $L$ given by (also see Kotsovinos and List 1977 and Baker 1980)

$$
L=M_{o}^{3 / 4} / F_{o}^{1 / 2}
$$

where $\rho M_{0}$ and $\rho F_{o}$ are the rates of addition of momentum and buoyancy at the source.

George et al. (1977) measured the mean and turbulence quantities using hot-wire anemometry with modern digital techniques. They used a two wire probe to measure the temperature and velocity. The results were substantially different from those measured by Rouse et al. (1952). It was also found that about $15 \%$ of the total vertical heat transport was contributed by turbulence. Nakagome and Hirata (1975) also made similar measurements in an independent effort, but obtained lower values of temperature and velocity intensities.

Beuther (1980, see also Beuther and George 1982) used a cross-wire to measure up to fourth moments for an axisymmetric plume in a stably stratified environment. He found that the stable stratification made the mean velocity and buoyancy profiles narrower. Ogino et al. (1980) used hot films and thermocouples to measure the centerline decay rates of velocity and temperature in round buoyant jets in both the neutral and stratified environments. Shabbir and George $(1985,1987)$ reported hot wire measurements for a round plume. Papanicolaou and List (1987) used thermistor probes to measure the temperature field in buoyant jets. Papanicolaou and List (1988) carried out a more comprehensive set of measurements using LDA and laser-induced fluorescence technique. They measured both the buoyant jet and the buoyant plume regions. Papantoniou and List (1989) reported results about the large structure of far field buoyant jets. 
Although existence of the similarity has been confirmed in these experiments, there has been some disagreement about the centerline values of the mean velocity and buoyancy profiles as well as the plume spreading rate. Chen and Rodi (1980) (see Rodi 1986 for an updated review) reviewed the existing experimental data on plumes and recommended profiles which were very close to those of George et al. (1977). List (1982) in his review suggested that all the measurements in the axisymmetric geometry (except their's) have not been taken in the fully developed region. He also pointed out that none of these studies equated the integrated buoyancy flux with the source buoyancy flux. The buoyancy flux, $F$, which directly appears in the similarity relations was obtained in these studies by integrating the measured temperature and velocity profiles. This could result in some error since the probes used, hot wires, do not have the ability to resolve flow reversals believed to be present at the outer edges of the flow.

\subsection{Objectives of the Present Work}

From the above survey it is clear that there is a need of comprehensive data on neutral environment round plumes which can resolve the differences in the various experiments. Thus, one of the objectives of this study was to resolve whether, in fact, an axisymmetric plume experiment which conserves buoyancy could be carried out. Since for an ideal gas in a neutral environment the conservation of energy also implies the conservation of buoyancy, (see Chen and Rodi 1980) the energy and buoyancy integrals should equal the values for these quantities at the source. In the present experiment source conditions were carefully monitored to obtain the rate at which buoyancy was added at the source. The two wire probe measurements were carried out to achieve this first objective since the single hot-wire (the second wire of the probe was operated as a cold wire) involves smaller error for the velocity measurement than the $x$-wire probe. The second objective of the study was to provide more detailed measurements which can shed some light on the structure of turbulence in plumes and help identify the affect of buoyancy on turbulence. 
For this purpose a three wire probe was used to measure the temperature and the two velocity components. This allowed the measurement of the various moments up to third order which provide information about the turbulent transport of momentum and thermal energy. The budgets of the mean momentum and energy differential equations are carried out to see how do the various terms in these equations balance out. Furthermore the balances of the turbulence kinetic energy and temperature variance differential equations are also carried out. The rates of mechanical and thermal dissipation are obtained as the closing entries in these balances. These balances show which physical phenomena dominate the different regions of the flow. Turbulence kinetic energy budget can be used to find the direct influence of buoyancy on turbulence by looking at the buoyancy production term.

\section{Analytics}

\subsection{Governing Equations}

The coordinate system for analyzing the round buoyant plume is sketched in Figure 1. The vertical velocity and direction are represented by $W$ and $z$ respectively while $U$ and $r$ denote the corresponding radial values. The gravitational vector is chosen to correspond with the negative $z$-direction.

The mean continuity, momentum, and energy equations, within the Boussinesq approximation and after neglecting the viscous diffusion (justified for high Reynolds number flow), are given by

$$
\begin{aligned}
\frac{1}{r} \frac{\partial r U}{\partial r}+\frac{\partial W}{\partial z} & =0 \\
U \frac{\partial W}{\partial r}+W \frac{\partial W}{\partial z} & =-\frac{1}{r} \frac{\partial}{\partial r}(r \overline{u w})-\frac{\partial}{\partial z}\left(\overline{w^{2}}-\overline{u^{2}}\right)+g \beta \Delta T, \\
U \frac{\partial}{\partial r}(g \beta \Delta T)+W \frac{\partial}{\partial z}(g \beta \Delta T) & =-\frac{1}{r} \frac{\partial}{\partial r}(r g \beta \overline{u t})-\frac{\partial}{\partial z}(g \beta \overline{w t}) .
\end{aligned}
$$

The radial momentum equation has been used to replace $\frac{-1}{\rho} \frac{\theta P}{\theta z}$ in the original vertical momentum equation by $\frac{\partial}{\partial z} \overline{u^{2}}$. (This integration actually results in $\frac{1}{\rho} \frac{\partial P}{\theta z}=\frac{\theta}{\partial z}\left\{\left(\overline{u^{2}}+\overline{v^{2}}\right) / 2\right\}$ 
but the assumption of $\overline{u^{2}}=\overline{v^{2}}$ has been used to obtain the final result.) In writing down the energy equation it was assumed that the ambient temperature is constant so that the local temperature $T$ was replaced by the local buoyancy $g \beta\left(T-T_{\infty}\right)=g \beta \Delta T$.

The similarity relations for the mean buoyancy and velocity were given by Batchelor (1954). For the axisymmetric geometry the similarity form of the variables is given by

$$
\begin{array}{r}
\eta=r / z, \quad g \beta \Delta T=T_{s} t(\eta), \quad W=W_{s} f(\eta) \\
W_{s}=F^{1 / 3} z^{-1 / 3}, \quad T_{s}=F^{2 / 3} z^{-5 / 3}
\end{array}
$$

The other moments involving temperature and velocity scale in a similar fashion e.g. $g \beta \overline{w t}=W_{s} T, h_{1}(\eta)$ and $\overline{u w}=W_{s}^{2} r_{1}(\eta)$.

Temperature variance and turbulence kinetic energy equations will be needed later on. Their derivation can be found in standard texts on turbulence and here their final form is given. The temperature variance equation for a round plume is

$$
U \frac{\partial}{\partial r}\left(\frac{\overline{t^{2}}}{2}\right)+W \frac{\partial}{\partial z}\left(\frac{\overline{t^{2}}}{2}\right)=-\frac{1}{2}\left\{\frac{1}{r} \frac{\partial}{\partial r}\left(r \overline{u t^{2}}\right)+\frac{\partial}{\partial z}\left(\overline{w t^{2}}\right)\right\}-\overline{u t} \frac{\partial T}{\partial r}-\overline{w t} \frac{\partial T}{\partial z}-\epsilon_{t}
$$

The above equation states that the advection of temperature variance is balanced by the turbulent diffusion of the temperature fluctuations; the turbulence production due to mean temperature gradients; and the molecular dissipation. The diffusion term merely redistributes energy from one point in the flow field to another. The gradient production term transfers energy from the mean flow to turbulence. And finally the dissipation term represents the rate of dissipation of energy into heat through molecular action and represents a loss of energy to turbulence.

The turbulence kinetic energy equation for a buoyant plume is

$$
\begin{aligned}
U \frac{\partial}{\partial r}\left(\frac{\overline{q^{2}}}{2}\right)+W \frac{\partial}{\partial z}\left(\frac{\overline{q^{2}}}{2}\right)= & -\frac{1}{2}\left\{\frac{1}{r} \frac{\partial}{\partial r} r\left(\overline{u q^{2}}+\overline{u p} / \rho\right)+\frac{\partial}{\partial z}\left(\overline{w q^{2}}+a \overline{w p} / \rho\right)\right\} \\
& -\overline{u^{2}}\left(\frac{\partial U}{\partial r}+\frac{U}{r}\right)-\overline{u w} \frac{\partial U}{\partial z}-\overline{u w} \frac{\partial W}{\partial r}-\overline{w^{2}} \frac{\partial W}{\partial z}+g \beta \overline{w t}-\epsilon
\end{aligned}
$$


The various terms in the above equation have the same physical meaning as the corresponding terms in the temperature variance equation.

All the terms in these equations, except the dissipation rates, were measured directly. The axisymmetry assumptions of $\overline{u^{2}}=\overline{v^{2}}, \overline{w v^{2}} \approx \overline{w u^{2}}$ were used in carrying out the budget of equation (11) and were verified by rotating the $x$-wire by ninety degrees in preliminary measurements. The mechanical and thermal dissipation rates will be obtained as the closing terms in the balances of these equations.

Since at present there is no method of measuring the pressure diffusion terms it was decided to make an estimate of these. For this purpose the model of Lumley (1978) was used, which gives $\overline{p u_{i}}=-\overline{q^{2} u_{i}} / 5$. It is realized that there is no experimental verification of the above relation, but at the same time it is believed that it is better to use this estimate rather than just setting the pressure diffusion equal to zero. This also means that the term labelled as "dissipation", to be shown later in the kinetic energy balance, is really the sum of the dissipation rate, the errors due to the above assumption and the measurement errors present in the other terms of the kinetic energy equation.

\subsection{Integral Constraints for Neutral Environment}

The energy equation (8) can be integrated across the flow to obtain

$$
F=2 \pi \int_{0}^{\infty} g \beta(W \Delta T+\overline{w t}) r d r=\text { constant }=F_{o}
$$

where $F_{o}$ is the rate at which buoyancy is added at the source. The second term inside the integral is the turbulent contribution to the total buoyancy flux. This integral constraint implies that for an ideal gas plume, in a neutral environment, both the energy (enthalpy) and the buoyancy are conserved. This integral constraint must be satisfied by any experiment to within a certain experimental uncertainty. It will be shown in section 4.5 that the present experiment satisfies this constraint to within $10 \%$. 
The momentum equation (7) can also be integrated across the flow to obtain

$$
\frac{d}{d z}\left\{2 \pi \int_{0}^{\infty}\left[W^{2}+\overline{w^{2}}-\overline{u^{2}}\right] r d r\right\}=2 \pi \int_{0}^{\infty}(g \beta \Delta T) r d r
$$

where the contribution due to turbulence has been retained. For later use the above equation is non-dimensionalized by the source momentum flux $M_{0}$ and the length scale $L$, given by equation (5), to obtain

$$
\frac{d}{d \xi}\left\{\frac{2 \pi}{M_{\mathrm{o}}} \int_{0}^{\infty}\left[W^{2}+\overline{w^{2}}-\overline{u^{2}}\right] r d r\right\}=2 \pi \frac{L}{M_{\mathrm{o}}} \int_{0}^{\infty}(g \beta \Delta T) r d r
$$

The above equation shows that the momentum flux changes with vertical distance. Again it will be shown that the present experiment satisfies this constraint within experimental error.

\subsection{Stratification of the Ambient}

If the ambient temperature $T_{\infty}$ changes with height (i.e. $T_{\infty}=T_{\infty}(z)$ ), then equation (8), becomes

$$
U \frac{\partial}{\partial r} g \beta \Delta T+W \frac{\partial}{\partial z} g \beta \Delta T=-\frac{1}{r} \frac{\partial}{\partial r}(r g \beta \overline{u t})-\frac{\partial}{\partial z}(g \beta \overline{w t})-W \frac{d}{d z}\left(g \beta T_{\infty}\right) .
$$

The extra term $W \frac{d}{d z}\left(g \beta T_{\infty}\right)$ arises due to the ambient stratification (see e.g. Batchelor 1954). Integrating the above relation gives the following integral constraint

$$
\frac{d}{d z}\left[2 \pi \int_{0}^{\infty} g \beta(W \Delta T+\overline{w t}) r d r\right]=-g \beta \frac{d T_{\infty}}{d z} 2 \pi \int_{0}^{\infty} W r d r .
$$

We immediately see that for the neutral environment the right hand side is zero and the above relation reduces to equation (12). On the other hand, if $\frac{d T_{\infty}}{d z}$ is not zero, the entrainment of progressively lighter or heavier fluid (as measured by the integral on the right hand side) decreases or increases the buoyancy.

In laboratory simulations of plumes some degree of stratification is always present. This is because of the finite size of the facilities and the fact that length of such experiments is 
several hours ( $\approx 10$ hours). As a consequence, an inversion layer builds near the ceiling and may or may not reach an equilibrium height above the zone of measurement.

Equation (16) provides us with a criterion to determine if a particular ambient must be regarded as stratified. Multiplying equation (16) by $z / F_{0}$, in order to make it dimensionless, we have

$$
\frac{z}{F_{o}} \frac{d F}{d z}=-\frac{z}{F_{o}} g \beta \frac{d T_{\infty}}{d z} 2 \pi \int_{0}^{\infty} W r d r
$$

For a neutral environment $\frac{z}{F_{0}} \frac{d F}{d z}=0$. For a laboratory simulation of a neutral environment

we must have $\frac{z}{F_{0}} \frac{d F}{d z}<<<1$ to insure buoyancy integral is nearly independent of height as required for the neutral case. In section 4.2 it will be shown that the above criterion is satisfied for the present experiment. (Note that this criterion is in addition to the requirement that $F=F_{\text {o. }}$ )

\section{Experiment}

\subsection{Facility}

The plume generator is the same as that used by George et al. (1977) and by Beuther and George (1982). The heat source consisted of two sintered bronze discs which were heated with eight electric heaters (1500 watt Watlow fire cartridges). Air from a compressed air line was first passed through these heated discs, and then through a set of screens, before finally exiting through a 12:1 contraction ratio nozzle. This resulted in an exit profile which was uniform outside the wall boundary layer to within $2 \%$. The turbulence intensity at the exit was typically $0.5 \%$. The space between the outer casing and the nozzle assembly was filled with silica gel for insulation purposes.

A feedback thermocouple, placed at the nozzle exit, was connected to an Electromax temperature controller. Under typical operating conditions, about two hours were required for the generator to reach thermal equilibrium. However, data collection was not started 
until after the plume had been running for about four hours. The variation in the exit temperature was \pm 1 degree Celsius over this period compared to the nominal operating value of 295 degree Celsius.

The plume facility is schematically shown in Figure 2. A square screen enclosure was placed around the plume which was $2 \mathrm{~m} \mathrm{X} 2 \mathrm{~m}$ in cross section and 5 meter in height. Thus the enclosure was far enough from the flow to present any hindrance to it.

During the course of this study it was also discovered that placing small circular screens around the source could significantly influence the buoyancy and velocity profiles and can act as a source of discrepancy between the various investigations. (The details of this issue are given in Appendix B.) Such screens are employed in order to prevent the flow from deflecting or moving off-center. No such screens were used in the present study.

The dimensions of the room housing the facility were $6 \mathrm{mX6m} \mathrm{X} 10 \mathrm{~m}$ and it was completely shut off for the duration of the experiment. This was done in order to prevent the flow from being disturbed from cross drafts which might arise from the HVAC system. A flow visualization study was also carried out to confirm that there was no plume drift.

\subsection{Instrumentation}

Two kinds of hot wire probes were used to measure the velocity and temperature fields. The first was a two wire parallel probe (DANTEC 55P76). The leading wire was used as a cold wire to measure the temperature. It had a diameter of $1 \mu m$ and a sensitive wire length of $1.2 \mathrm{~mm}$. It was operated in the constant current mode with a heating current of 0.3 milliamperes to insure that its velocity response was negligible. The bottom wire was used in the constant temperature mode to measure velocity. The sensor had an overall length of $3 \mathrm{~mm}$. It was etched at the center to give an effective length of $1.25 \mathrm{~mm}$ and had a diameter of $5 \mu \mathrm{m}$. In order to get an optimum response for both the velocity and 
the temperature from this wire, an overheat ratio of 0.4 was used.

The second probe used was a combination of a cross-wire and a temperature wire. The temperature wire was again $1 \mu \mathrm{m}$ in diameter and was heated with a current of 0.15 milliamperes. The $\mathrm{x}$-wire was made of $5 \mu \mathrm{m}$ gold-plated wire and had an $1 / \mathrm{d}$ of 125 . All the velocity sensors were operated in the constant temperature mode using DANTEC 55M anemometer systems. For the temperature wire the 55M20 bridge was used with the 55M01 system.

To check the axisymmetry of the flow and to locate the plume center, an array of sixteen thermocouples was used which were arranged in a $4 \times 4$ grid. The scanner used for acquiring data from these thermocouples was made using four millivolt conditioners manufactured by Analog Devices (Model 2B54A). These conditioners were specially designed for thermocouple applications. A gain of 1000 was employed so that the millivolt signal from the thermocouples was converted into volts. The scanner was driven by TTL logic and was interfaced with a PDP 11/34 mini-computer. The output from the scanner was sampled and digitized using a 16 bit A/D converter. The scanner permitted taking as many as 400 samples per second. However, the fastest rate used in the experiments was 32 samples per second. All the thermocouples used in the experiment were Copper-Constantan and were ice referenced. The outputs from all the anemometers and signal conditioners were digitized by an 16 bit $A / D$ converter interfaced with the PDP $11 / 34$.

The integral time scale of the flow was estimated to be 0.2 seconds. This implied that the data sampling rate could not be greater than 5 samples per second since the additional samples would not contribute to the statistical convergence. Therefore, the sampling rate was set to 4 samples per second. For each data point 2048 samples were taken and this gave a total averaging time of about 17 minutes per data point. For a Gaussian signal this corresponds to a relative error of $1 \%$ for the mean values. 


\subsection{Calibration Schemes}

The constant current wire gave a linear response to the temperature changes. Since for the velocity wire the output voltage was a function of both the velocity and the temperature it was handled by expressing the wire Nusselt number, $N u$, as a function of the Reynolds number. In particular

$$
R e=\sum_{n=0}^{n=4} A_{n} N u^{n / 2} .
$$

The coefficients $A_{n}$ are temperature independent; the temperature dependence enters through the Nusselt number. For angle response of the $\mathrm{x}$-wire a Hinze-type relation was used which incorporated a velocity- dependent $\mathrm{k}$-factor i.e.

$$
\frac{U_{e f f}}{U_{0}}=\left[\cos ^{2} \phi+k^{2}\left(U_{0}\right) \sin ^{2} \phi\right]^{1 / 2},
$$

where $U_{\text {eff }}$ is the effective cooling velocity, $U_{o}$ is the flow velocity, and $\phi$ is the angle between the flow velocity and the normal to the wire. For details of these calibrations see George et al. (1989) and Shabbir et al. (1990).

The calibration of hot wires was carried out at the exit of the plume generator where it was possible to obtain the desired velocities and temperatures. It was found to be more efficient to first heat the plume generator to a particular temperature, and then vary the velocity starting from a low velocity and then gradually increasing it. Whenever velocity and temperature were changed, sufficient time was provided for the plume generator to reach thermal equilibrium. The reference temperature was obtained with a CopperConstantan thermocouple placed at the source and the reference velocity was obtained by doing the mass balance on a rotameter in the inlet line which in turn had been calibrated using LDA and wet-meters.

Calibration of the wires was done both before and after the experiment. Normally the velocity calibrations were found to be very stable. However the temperature wire was 
sometimes found to drift appreciably, presumably related to the stability of the DANTEC 55M20 bridge. All such data was discarded.

\subsection{Errors in Measurements}

There are three primary sources of error in a $\mathrm{x}$-wire signal at low velocities: rectification; cross-flow; and lack of directional sensitivity when the flow velocity makes larger inclination angle with the wire normal. The problem of rectification is obvious for a single wire in which the flow must reverse its direction for rectification to occur. Tutu and Chevray (1975) have pointed out that rectification errors are more subtle and serious for $x$-wires than for single wires. They also showed that the combined effects of rectification and cross-flow lead to the under-estimation of the second and higher moments.

An additional manifestation of the rectification phenomenon is the occurrence of voltage pairs which could not be resolved into velocity pairs from the angle calibration. In other words, the instantaneous voltage pairs obtained do not lie in the calibrated region and can not be inverted by equation (19). For such data, the word "dropout" is probably a more accurate description than "rectification". Dropout is usually caused by a high intensity in the $u$ or $v$ component and is especially troublesome when the mean velocity is low. As noted by Beuther (1980) "This is because wires are fairly insensitive to direction at low velocities and any small measurement error (electronic noise, prong support interference, velocity component perpendicular to the $\mathrm{x}$-wire plane, wake of one wire on the other, or a velocity or temperature gradient between the wires) can create a large error in the output". The dropout was small in the central core of the plume but was as big as $40 \%$ at it's outer edges.

Since the dropped data points are associated with the low velocities, the dropout would tend to bias the velocity statistics toward the higher side. It should also be noted that like the dropout, the flow reversals on the wire are also associated with low mean velocities. It is 
very difficult to quantify the combined effects of all, these errors in the current experiment. As a word of caution it is recommended that all the third moments and the second moment balances must be regarded as qualitative in nature rather than as quantitative beyond about $\eta \approx 0.15$ or so.

\section{Results}

\subsection{Source Conditions}

An accurate knowledge of the source conditions is essential to check whether or not the experiment conserves buoyancy. Also the rate at which buoyancy is added at the source appears in all the similarity relations. In view of the fact that plumes in stratified environments also satisfy similarity relationships using the local buoyancy integral, it is more accurate to use the source buoyancy (which must be conserved for the neutral environment) than the one obtained by integrating the measured velocity and temperature profiles.

Schlieren visualization showed that the flow was not laminar after about one diameter. Source conditions for the single wire and $x$-wire experiments are given in Table I and these differ with each other slightly. Since both the momentum and buoyancy are added at the source, the flow near the source is more like a buoyant jet and not a plume. However, as the flow evolves, the buoyancy overwhelms the momentum added at the source and after a certain distance from the source the flow is governed by buoyancy alone. How far away from the source this happens is determined by buoyancy and momentum added at the source (characterized by the length scale $L=M_{0}^{3 / 4} / F_{0}^{1 / 2}$ ).

It is believed that the buoyant jet reaches an asymptotic plume like condition for $\xi(=$ $z / L)>5$. For the source conditions of this study, this criteria is met as $\xi$ ranges from 6.5 to 15. The centerline values of buoyancy, non-dimensionalized by $M_{0}^{1 / 2} / F_{0}$, (and represented by $t(\eta, \xi))$ can be plotted as a function of $\xi$. For a buoyant jet these should follow a 
horizontal line and for a buoyant plume these should follow a $-2 / 3$ slope (see Morton 1959, Kotsovinos and List 1977, Baker 1980). Figure 3 shows such a plot for the present experiments and clearly these correspond to a plume like flow. Note that for some of the locations centerline buoyancy was not measured and for such cases either the next point off the centerline axis was used or the profile was extrapolated to the centerline axis. Because of this there is some scatter in the data points shown in figure 3.

\subsection{Ambient Conditions}

Beuther and George (1982) and Beuther (1980) have shown that a small stratification of the ambient fluid can cause a significant loss or gain of buoyancy depending on the sign of the right hand side of equation (16) and hence can appreciably change the shape of the profiles. In the experiment of Beuther (1980) the ambient was stably stratified and the data collapsed in the similarity variables when the local value of the buoyancy flux was used. His measurements showed that the stable stratification made the mean profiles narrower and higher.

None of the studies reported in the literature have monitored or documented the ambient temperature changes with the exception of Ogino et al. (1980) and Kotsovinos (1985). In the present experiment the ambient was continuously monitored by using seven CopperConstantan thermocouples placed at different heights. Figure (4) shows the change in ambient temperature both as a function of height and time for one of the experimental runs. It is seen that for the first two hours of operation a significant change in the temperature occurs. However, after this it changes very little, especially over the heights where the measurements were taken.

According to the criteria established in section 2.3 for avoiding stratification effects,

$$
-\frac{z}{F_{o}} g \beta \frac{d T_{\infty}}{d z} 2 \pi \int_{0}^{\infty} W r d r<<1 .
$$

For this experiment the value of the above relation is 0.003 which is very small as compared 
to 1. For the experiment of Beuther (1980) the value of the above relation was about 0.36 . Based on these we can confidently say that the measurements reported herein are taken in the neutral environment.

\subsection{Mean Flow Results}

The mean buoyancy profile is shown in figure 5 . The closed symbols refer to the two wire probe measurements and the open symbols refer to the three wire probe measurements. The curve shown is the Gaussian fit to data and is given by

$$
t(\eta)=9.4 \exp \left(-68 \eta^{2}\right)
$$

The data collapse is very reasonable, and gives confidence that the plume is fully developed at the locations where the measurements are taken. The centerline value of 9.4 is slightly higher than the value of 9.1 recommended by George et al. (1977) who used the same instrumentation but calculated the buoyancy flux by integrating the measured velocity and temperature profiles. If like them the present data is normalized by the local buoyancy flux, then the centerline value of the mean buoyancy will be 9.0 thus indicating that the differences are attributable to errors in their calculation of buoyancy. The centerline value reported here is considerably lower than the $A_{t}=11.0$ reported by Rouse et al. (1952). Most of this difference can be attributed to the fact that they did not include the turbulence contribution to the total heat flux which can amount to as much as $18 \%$ (to be discussed later in section 4.5). If this is taken into account, then their value of $A_{t}$ would drop from 11.0 to 9.9 which is only $5.3 \%$ larger than what is reported here. Ogino et al. (1980) measured $A_{t}=9.4$ which is the same value as reported here whereas Kotsovinos (1985) reported a value of 8.8. On the other hand Papanicolaou and List (1987) and (1988) reported values of 11.11 and 14.28 respectively; the former measured with thermistor probes and the later using the LIF technique. The value recommended by Chen and Rodi (1980) and Rodi (1986) is 9.35 which is in excellent agreement with the present experiment. 
Half width of the buoyancy profile is 0.101 . As can be seen from Table II this is in excellent agreement with the recommendations of George et al. (1977), Chen and Rodi (1980), and Rodi (1986). It is approximately the same as suggested by the measurements of Rouse et al. (1952) and Kotsovinos (1985) but is about $7.8 \%$ larger than the value reported by Papanicolaou and List $(1987,1988)$.

The buoyancy profile measured using thermocouples is shown in figure 6. It has the same centerline value and half width as the hot wire measured one. This not only complements the hot wire measurements, but also shows that the slight differences between the present study and that of Rouse et al. (1952) are not instrumentation related. However, there are still substantial differences between the results of this study and Papanicolaou and List $(1987,1988)$; their profiles are higher and narrower then what is measured in the present experiment.

The mean velocity profile is shown in figure 7 , the curve is the Gaussian fit to the data and is given by

$$
f(\eta)=3.4 \exp \left(-58 \eta^{2}\right)
$$

The centerline value of 3.4 is exactly the same as reported by George et al. (1977) and Ogino et al. (1980). Note that the velocity is less sensitive to the method of evaluating the buoyancy integral than is the temperature since it varies as $F^{-1 / 3}$ versus $F^{-2 / 3}$. The value recommended by Chen and Rodi (1980) and Rodi (1986) is 3.5. As can be seen from Table II the values reported by Rouse et al. (1954), Nakagome and Hirata (1975), and Papanicolaou and List (1988) are somewhat higher.

The half width for the velocity, $b_{w}$, is 0.1075 and is in excellent agreement with the findings of George et al. (1977), Chen and Rodi (1980), and Rodi (1986). We note that the velocity profile is wider then the buoyancy profile. This is also in agreement with the findings of Nakagome and Hirata (1975) and George et al. (1977) and the 
recommendations of Chen and Rodi (1980). The measurements of Rouse et al. (1952) and Papanicolaou and List (1988) show just the opposite.

The measured radial velocity profile is also shown in figure (7). This can also be obtained from the continuity equation as

$$
U=\frac{1}{r} \int_{0}^{r} \frac{\partial W}{\partial z} r d r
$$

If the above equation is cast into similarity variables and if the Gaussian expression is used for the vertical velocity (i.e. equation 21) we obtain the following expression for the mean radial velocity

$$
l(\eta)=\left(\frac{-5}{3}\right)\left(\frac{1}{2 \alpha}\right)\left[\frac{f(0)-f(\eta)}{\eta}\right]+\eta f(\eta) .
$$

where $\alpha$ is the constant appearing in the Gaussian fit and for the present experiment is given in equation (21). From figure (7) we note that the mean radial velocity is very small as compared to the mean vertical velocity, as is assumed when making the thin shear layer approximation for such a flow. Also note that the continuity inferred radial velocity becomes negative (directed inwards) after about $\eta=0.10$ whereas measured one does not. The difference can be attributed to the small values of $U$ (which are of order $\delta / L$ ) and the hot wire cross-flow errors which are of the same order.

\subsection{Turbulence Properties}

Various second moments involving temperature and velocity are shown in figures 9 through 13. All these moments are plotted in the similarity variables because that is the way these appear in the equations of motion. When comparing the statistics of different flows with each other (e.g. jets versus plumes) it is sometimes more helpful to use turbulence intensities and for this reason the centerline and the peak values of these will also be mentioned. The similarity profile of temperature variance has a centerline value of 12.5 and has a slight off axis peak which corresponds to a temperature intensity of about $40 \%$. This value is in good agreement with the values of $43 \%$ and $44 \%$ reported by Nikagome 
and Hirata (1975) and Kotsovinos (1985) respectively. The measurements of Papanicolaou and List (1988) show a peak value of $42 \%$. However, due to substantial differences in the mean buoyancy profile between this reference and the present study, this intensity is a misleading parameter for comparison. If their concentration profile is translated into similarity variables it gives a centerline value of about 32.0 which is about three times higher than the value measured here; a very large difference. So there are substantial differences between the present study and that of Papanicolaou and List (1988) for both the mean and the fluctuating buoyancy measurements. These differences are perplexing because there are no hot-wire errors involved in the measurement of temperature.

The centerline value of the vertical turbulent heat flux, $\overline{w t}$, is 1.85 . Measurements of George et al. (1977) correspond to a value of 1.90 whereas those of Papanicolaou and List (1988) correspond to a peak value of 3.0. It can be seen from figure 9 that for $\eta>0.16$ the $x$-wire measurements are almost a factor of two higher than the single wire measurements. This error is due to the drop out and poor directional response associated with $\mathrm{x}$-wires at higher turbulence intensities as was discussed in section 3.4. This kind of error can be seen in some of the other second moments as well. Obviously wherever available, single wire results are to be preferred. The correlation coefficient $\overline{w t} / w^{\prime} t^{\prime}$ has a peak value of 0.7 at $\eta=0.12$. It confirms the value of 0.67 measured by George et al. (1977) and, as suggested by them, such a strong correlation between the turbulent velocity and temperature field is to be expected for a flow in which buoyancy initiates the velocity field which in turn influences the buoyancy field. Nakagome and Hirata (1975) reported a value of 0.46 for this correlation. The measurements of Papanicolaou and List $(1987,1988)$ give a peak value of about 0.51 . The radial turbulent heat flux $\overline{u t}$, shown in figure 10 , has a peak value of 0.90 at $\eta=0.07$ and its shape is very close to the derivative of the mean buoyancy.

The centerline value of vertical velocity fluctuations $\overline{w^{2}}$ (figure 11) is 1.1. This corresponds to a turbulent intensity of about $32 \%$ which is slightly higher than the value of $28 \%$ 
measured by George et al. (1977). The value reported by Papanicolaou and List (1988) is $25 \%$. They reported a centerline value of the mean vertical velocity of 3.85 as compared to 3.4 measured in the present investigation and this might explain the differences. If their results are translated to the similarity variables one obtains $\overline{w^{2}}=0.90$ which is close to what is measured here. The centerline value of the radial velocity fluctuations $\overline{u^{2}}$ is 0.65 and corresponds to a turbulence intensity (i.e. $\dot{u} / W$ ) of $19 \%$. The value reported by $\mathrm{Pa}$ panicolaou and List (1988) is about $15 \%$. The shear stress profile is plotted in figure (13) and has a maximum value of 0.32 at $\eta=0.07$ and its shape strongly resembles the mean velocity gradient. If normalized by the centerline value of mean vertical velocity, the peak value of shear stress is about 0.09 . It should be noted that both shear stress and radial heat flux reported here have small finite values at the center rather than being zero. This is due to the finite values of the various hot wire errors for these quantities.

The foregoing results can be used to calculate the turbulent Reynolds number (see Townsend 1956) $R E_{T}=W(0) l_{u / 2} / \nu_{T}$, where $W(0)$ is the centerline value of mean vertical velocity, $l_{u / 2}$ is the velocity half width, and $\nu_{T}$ is turbulent eddy viscosity defined by

$$
\nu_{T}=-\frac{\overline{u w}}{\frac{\partial W}{\partial r}} .
$$

Current measurement show that $R E_{T}$ has an average value of 22.5 . For round wake and jet these values are 14.1 and 32 respectively (Townsend 1956). Lumley (1971) had calculated this value for a plume to be 14 from the measurements of Rouse et al. (1952) which is very close to the wake value. The reason given by Lumley (1971) for this plume value being closer to wake then jet was that in plumes the buoyancy causes the lateral squeezing of the eddies which makes them structurally closer to wake. The current experiment shows that for a plume this value is in between the wake and the jet and, therefore, plume can not be considered structurally closer to a wake. However, the observation of Lumley (1971) regarding the lateral squeezing of eddies in plumes still holds because the plume value is smaller then the jet value. The turbulent thermal diffusivity can also be calculated by 
using

$$
\gamma_{T}=-\frac{\overline{u t}}{\frac{\partial T}{\partial r}} .
$$

and the result can be expressed in the form of a turbulent Prandtl number $\nu_{T} / \gamma_{T}$. In the present experiment this value ranges from 0.7 to 1.0 with an average value of 0.85 .

The third order moments for the last four measurement stations are shown in figures 14 and 15. Some of these third moments will be used in balancing the temperature variance and turbulence kinetic energy equations. The moments $\overline{w t^{2}}, \overline{w^{2} t}$ and $\overline{t^{3}}$ all start from finite values at the center and then show a slight off-axis peak before rolling off to small values. The moments $\overline{w^{2} u}, \overline{w u^{2}}$ and $\overline{u t^{2}}$ show negative values near the centerline axis. Some of the third moments show small values at the centerline rather than being zero due to the cross-flow errors associated with the hot-wires. The curves shown in these figures are the recommended fits to the data and these are tabulated in Table A-I along with the fits to the higher moments.

One disappointing feature of these third moments is their wide scatter. For the number of samples taken, the relative error in these is estimated to be about $10 \%$ near the center line. This value increases away from the core as the turbulence intensity increases. It should also be noted that such scatter is also present in the other plume experiments which have been cited earlier in this paper.

\subsection{Evaluation of the Integral Constraints}

As was discussed in section 2.2 the integral constraint arising from the energy equation implies that the buoyancy flux should be conserved for a plume in a neutral environment. Therefore, the local values of buoyancy flux, $F$, at different heights were obtained by using equation (12). Note that this integration includes the turbulent contribution as well. Figure 16 shows the ratio of this local buoyancy to the source buoyancy, $F / F_{0}$, at various heights. It is clear that the experiment conserves buoyancy within $10 \%$ which is within 
the experimental error. The turbulent contribution was found to be in the range of $15 \%$ to $18 \%$ of the total buoyancy flux. This is a substantial percentage and can not be ignored in any integral type analysis of this flow. This was noted by George et al. (1977) who had also found this contribution to be about $15 \%$ and subsequent studies by Beuther (1980), Shabbir and George (1985) and Papanicolaou and List (1988) had confirmed this. It should be noted that the accuracy of the integration is better than might have been expected from a consideration of the hot wire errors at large radius. This is because the temperature and velocity profiles fall rather sharply with radius so that the integrand of equation (12) is dominated by the profiles in the core region.

In order to check the integral momentum constraint, given by equation (14), the mean momentum flux, normalized by it's source value, is plotted against $\xi^{4 / 3}$ in figure 17 . It's derivative at a given location should equal the buoyancy acceleration at that location. The curve fit to the data gives $\frac{M}{M_{0}}=0.34 \xi^{4 / 3}$. This can be differentiated to obtain the left hand side of equation (14). The results are given in Table III and the agreement between the two sides of the momentum integral (equation 14) is satisfactory.

The turbulent contribution to the momentum flux is $2 \pi \int_{0}^{\infty}\left(\overline{w^{2}}-\overline{u^{2}}\right) r d r$ (see equation 13 ) and is found to be $4.6 \%$ of the mean momentum flux. This is quite small and, therefore, can be ignored in an integral type of analysis. Notice that both $\overline{w^{2}}$ and $\overline{u^{2}}$ are of the same order and if one is kept the other must also be retained. Papanicolaou and List (1988) kept only the $\overline{w^{2}}$ term in their analysis and thus erroneously concluded that in a plume the turbulent contribution to the momentum flux is about $15 \%$ of the mean.

\subsection{Balances of the Mean Energy and Momentum Equations}

The balance of the mean energy equation (equation 8 ) is shown in figure 18a. We observe that the radial advection term is extremely small as compared to the vertical advection term. This is because the mean radial velocity is negligible as compared to the vertical 
velocity. We also note that the vertical turbulent transport is extremely small as compared to the radial turbulent transport. Note that when this equation is integrated across the flow the radial advection and radial turbulent transport terms fall out. The term labelled as error represents the amount which is missing from the right hand side of equation (8). This error is due to the underestimation of the radial turbulent heat flux. We also observe that the relative magnitude of each term remains the same across the plume and that all the terms fall off to very small values after $\eta \approx 0.1$. In summary we can say that in a plume the mean flow advects the buoyancy in the vertical direction and turbulence transports it in the radial direction (figure $18 b$ ) i.e.

$$
W \frac{\partial}{\partial z}(g \beta \Delta T) \approx-\frac{1}{r} \frac{\partial}{\partial r}(r g \beta \overline{u t})
$$

The balance for the mean momentum differential equation (equation 7) is shown in figure 19a. We note that, like the mean energy equation, the advection in the radial direction is very small as compared to the advection in the vertical direction and that the turbulent transport of momentum in the vertical direction is negligible as compared to the turbulent transport in the radial direction. We also note that at $\eta=0.06$ the vertical advection changes its sign and after $\eta \approx 0.11$ advection and turbulent transport have exchanged sides in the balance as is depicted in figure $19 \mathrm{~b}$. The body force, due to gravitational convection, is the second largest term in this budget. It is this term which is responsible for setting up the fluid in motion and links the mechanical and thermal flow fields. This term is also responsible for wider velocity profiles in plumes as compared to jets. We further note that the terms in the momentum budget do not fall off to small values after $\eta \approx 0.1$ as they did for the mean energy balance. We conclude that to the first order the vertical advection of momentum is balanced by the turbulence transport in the radial direction and the gravitational convection i.e.

$$
W \frac{\partial W}{\partial z} \approx-\frac{1}{r} \frac{\partial}{\partial r}(r \overline{u w})+g \beta \Delta T
$$


It should be noted that these balances are not trivial and that they further help to establish the consistency and accuracy of the experiment.

\subsection{Balances for the $\overline{t^{2}} / 2$ and $\overline{q^{2}} / 2$ Equations}

The balance of $\overline{t^{2}} / 2$ (equation 9 ) is shown in figure $20 \mathrm{a}$. We note that advection term forms a major portion of the budget in the central half of the flow before acquiring a small value after $\eta \approx 0.10$. Incidentally this location approximately corresponds to the temperature half width. At the centerline production of temperature variance is about half the magnitude of the advection but it monotonically increases until it peaks at $\eta \approx 0.07$. We note that the peak values of the advection and the production terms are about the same. The diffusion term is quite small as compared to the other terms. The thermal dissipation rate $\epsilon_{t}$ was not measured and, therefore, is obtained as the closing term in the budget and is the largest of all the terms. Obviously a successful computation of the temperature variance by any turbulence model is crucially dependent on how accurately this dissipation rate is calculated.

The budget suggests dividing the flow into two regions. In the inner region $(\eta<0.1$, which approximately corresponds to the temperature half width) advection and production mechanisms provide most of the energy to temperature variance and are balanced by the dissipation rate. Obviously the flow is not in local equilibrium (i.e. production $\neq$ dissipation) in this region. In the outer region $(\eta>0.1)$ the production of temperature variance is balanced by the dissipation rate and the flow in this region, therefore, can be approximated to be in local equilibrium. These regions are schematically depicted in figure $20 \mathrm{~b}$.

Figure 21 a shows that the turbulence kinetic energy balance and we note that the advection does not constitute as large a portion of the turbulence kinetic energy balance as it did for the temperature variance equation. After $\eta \approx 0.08$ it changes sign and 
becomes a loss to the budget. At the plume center the gradient production term is about one third of the advection and it increases until $\eta \approx 0.08$. Most of the contribution to this production is coming from the shear stress term, $-\overline{u w} \frac{\partial W}{\partial r}$, except near the plume center where $-\overline{w^{2}} \frac{\partial W}{\partial z}$ is dominant. The direct affect of buoyancy on turbulence is represented by the buoyancy production term and although it forms a substantial fraction of the balance, it is still about three times smaller than the the gradient production. Therefore, most of the turbulence is maintained by the gradient production and not by the buoyancy. This is in contrast to the mean velocity field where the affect of buoyancy force was much more stronger.

The rate of mechanical dissipation $\epsilon$ was not measured and is obtained as the closing term in the balance. Like the temperature variance, the turbulence kinetic energy budget can also be divided into two regions. In the outer region $(\eta>0.1)$ the mechanisms of production and dissipation rate approximately balance each other and the flow can be considered in local equilibrium. In the inner region $(\eta<0.1)$ this is not true and the situation is qualitatively sketched in figure $21 \mathrm{~b}$.

The velocity derivatives were not measured and, therefore, it is not known if the local isotropy existed in the present experiment. We can use the dissipation rate profile obtained above to calculate the turbulence Reynolds number $R E_{T}=\frac{u l}{\nu}=\frac{{\overline{q^{2}}}^{2}}{9 \epsilon \nu}$. The profile of this Reynolds number is shown in figure 22 for one representative height. The centerline value is about 1600 which may not be very large to insure the existence of local isotropy.

It should be cautioned that at the outer edges of the plume ( $\eta>0.15$ or so) these balances are more of qualitative nature than being quantitative because of the hot-wire errors. This is especially true for the dissipation rates which are obtained as the closing terms in these balances because any errors present in the measurement of other quantities will be lumped in to these. The largest error should be through the the third order moment 
(diffusion) terms. Since there are no errors involved in the temperature measurement, the temperature variance balance is more accurate than the turbulence kinetic energy balance.

\section{Further Remarks and Summary}

This paper reported a comprehensive set of measurements in a fully developed round turbulent buoyant plume. The source and the boundary conditions of the flow were carefully measured and documented. The measurements of the ambient temperature insured that the facility was not stratified and that the experiment corresponded to a plume in a neutral environment. Although a very simple and an easy aspect to measure, it is a very important part of the current experiment. The affect of stratification of the ambient fluid on the solutions admitted by the mean flow equations has been analyzed for some time (see e.g. Batchelor 1954) but its importance in measurement of laboratory plumes in neutral environments was first realized by Beuther (1980). His study showed that plumes are quite sensitive to the changes in ambient fluid temperature and what might otherwise be considered a slight stratification can produce large changes in the mean flow. As was mentioned in section 2.3 , this influence of the ambient stratification enters the mean en-

ergy equation through $g \beta W \frac{d T_{\infty}}{d z}$ term and Beuther's (1980) study showed that under such conditions of stratification this term makes up a considerable fraction of the mean energy budget. Since most of the experiments dealing with neutral environment do not monitor their ambients, this has been suggested as one of the probable reasons for the differences in plume experiments (Beuther and George 1982). Another source of ambient disturbance is weak co-flowing streams which are set up in the ambient fluid in order to compensate for the entrained ambient fluid when experiments are conducted in water tanks (see George 1990). Appendix B details one case of this sensitivity which can occur when small circular screens are placed around the plume source. It is shown that since the entrainment flow is driven by second order terms, a third order term can produce differences of a few percent. The findings of these studies and the experience in our laboratory shows that differences 
in the various plume experiments could be more due to the differences in their boundary conditions and less due to the differences in the instrumentation.

The present study confirmed some of the earlier measurements of mean flow variables. There are, however, substantial differences between the present study and that of $\mathrm{Pa}$ panicolaou and List (1988). In order to explore the possible reasons for the differences between the present results and those of the Papanicolaou and List (1988), lets look at the temperature field measurements i.e. the mean buoyancy and temperature variance (mean buoyancy and concentration variance in the experiment of Papanicolaou and List 1988). We choose these quantities because there are no hot-wire errors involved in their measurement. Because of this fact it is very puzzling to see that the present results and those of Papanicolaou and List (1988) exhibit large differences. It should be noted that the local total tracer flux in the experiments of Papanicolaou and List (1988) is much larger than its source value, rather than being equal to it (within certain experimental uncertainty). Papanicolaou and List (1988) argue that the reason for this was a systematic error in their mean concentration measurements and that when the mean temperature decay rates from their earlier investigation (Papanicolaou and List 1987) are employed then the total tracer flux is conserved. However, this line of argument is weak in the sense that it still does not prove that their experiment conserved tracer flux. On the other hand the present experiment conserves buoyancy flux within $10 \%$.

It is very encouraging to note that the other studies which took care in insuring that their facilities were not stratified and were free of any ambient disturbances, obtained results which are very close to what is reported here. For example the centerline values of mean buoyancy and velocity measured by Ogino et al. (1980) and the present ones are exactly the same (they measured the centerline values only). The difference between the mean buoyancy profile measured here and that by Kotsovinos (1985) is only 6.5\%. The present study used both hot wire and thermocouples for the temperature measurement. 
The experiments of Ogino et al. (1980) and Kotsovinos (1985) employed thermocouples and thermistor probes respectively. These comparisons and facts give more credibility and confidence to the present measurements.

The current experiment conserves buoyancy within $10 \%$. It also satisfies the integral momentum constraint as the measurements show that the rate of increase of momentum with height is equal to, within experimental error, the buoyancy acceleration. These checks lend confidence to the measurements.

The turbulence measurements show that the peak turbulent intensities of the temperature and velocity are $40 \%$ and $32 \%$ respectively. The peak value of the vertical heat flux coefficient is found to be 0.7 . The present study only confirms the earlier results about these quantities and does not contribute anything new. The profiles of radial heat flux and shear stress were also measured. These are the most important of all the second moments appearing in the mean flow differential equations. This is the first time that these profiles have been reported for a plume and in this regard the current experiment has made a contribution to this area of turbulence.

The balances of the mean energy and momentum equation were also carried out and the the imbalance was shown as an error. This is also the first time that such a balance for a buoyant plume is carried out. The balance of the momentum equation clearly shows that the buoyancy force is as large as the transport by turbulent shear stress. This buoyancy force causes the velocity profiles in buoyant plumes to be wider and larger in magnitude then in jets. It then leads to increased production of turbulence energy through the gradient production term in the turbulence kinetic energy equation. This can be regarded as an indirect affect of buoyancy on turbulence.

The measurements allowed doing the balances of the temperature variance and turbu- 
lence kinetic energy. The thermal and mechanical dissipation rates were not measured and were obtained as the closing terms in these budgets. Despite certain measurement errors present in these budgets, which primarily emanate from the third moment terms, they provide useful information about the relative importance of the different phenomenon. It is found that although the direct affect of buoyancy on turbulence velocity field is substantial, most of the turbulence is still maintained by the gradient production. Therefore, it is concluded that in a buoyant plume the primary influence of buoyancy on turbulence is indirect, and enters through the mean velocity field (giving larger shear production). A contrast between the two budgets was the much larger magnitude of the advection of temperature variance as compared to the advection of turbulence kinetic energy.

It is cautioned that the moments involving velocity should be interpreted carefully toward the outer edges of the flow because of the larger hot-wire errors at these locations. This is especially true for the third moments. These moments and the temperature variance and turbulence kinetic energy budgets should be considered more as qualitative in nature rather than as quantitative beyond $\eta \approx 0.15$ or so. These errors can be avoided by measuring the flow with flying hot wire probes such as used by Capp et al. (1990) in their jet measurements. However, unlike jets, the velocities in laboratory plumes are very small in magnitude and a flight of probes through the flow will not only cause the plume to deflect or move off-center, but will also introduce undesirable air currents in an otherwise quiescent environment. This will necessitate a long wait between the probe traverses and will require a thorough probing of the facility for possible disturbances and stratification. The work in this direction is currently being evaluated by one of the authors (WKG). 
An earlier version of this work was presented at the Turbulent Shear Flow Conference TSF 87. We are grateful to $S$. Woodward and M. Kotas for their contributions to this study, and to late Professor C.B. Baker and Professor D.B. Taulbee for their encouragement. This work was initiated with the support of NSF Atmospheric Science, Meteorology Program Grant No. ATM80-23699A01 and was completed with the assistance of NSF Engineering, Fluid Mechanics Program Grant No. MSM83-16833. Final writing of paper was finished while the first author was in residence at ICOMP, NASA Lewis Research Center. 


\section{REFERENCES}

Baker, C.B. (1980) An Analysis of the Turbulent Buoyant Jet. PhD Dissertation, SUNY
at Buffalo, Buffalo, NY. Batchelor, G.K. (1954) Heat Convection and Buoyancy Effects in Fluids. Quart. J. Royal
Met. Soc. V.80, 339-358.

Beuther, P.D., S.P. Capp and W.K. George (1979) Momentum and Temperature Measurements in an Axisymmetric Turbulent Plume. ASME publication no. 79-HT-42.

Beuther, P.D. (1980) An Experimental Investigation of the Turbulent Buoyant Plume. PhD Dissertation, SUNY at Buffalo, Buffalo, NY.

Beuther, P.B. and George, W.K., (1982) The Turbulent Buoyant Plume in a Stratified Environment. Proc. of Nat. Cong. Theor. and Appl. Mech., Cornell Univ., Ithaca, NY

Capp,S.P., Hussein, H.J. and George, W.K. (1990) Velocity Measurements in a High Reynolds number Momentum Conserving Axisymmetric Turbulent Jet. Submitted to
JFM. Chen C. J. and W. Rodi (1980) Vertical Turbulent Buoyant Jets. Pergamon Press, New
York.

George, W.K., Jr., Alpert, R.L., Tamanini, F. (1977) Turbulence Measurements in an Axisymmetric Buoyant Plume. Int. J. Heat Mass Trans. 20, 1145-1154.

George, W.K., Beuther, P.D., and Shabbir, A. (1987) Polynomial Calibrations for Hot Wires in Thermally-Varying Flows. Expt. Thermal Fluid Science. 2,230-235. George, W.K. (1990) Governing Equations, Experiments, and the Experimentalist. Expt.
Thermal Fluid Science, 3, 557-566.

Kotsovinos, N.E. and E.J. List (1977) Turbulent Buoyant Jets. Part 1. Integral Properties.

Kotsovinos, N.E. (1985). Temperature Measurements in a Turbulent Round Plume. Int. J. Heat Mass Transfer, 28,4, 771-777.

List, E.J. and Imberger, J. (1973) Turbulent Entrainment in Buoyant Jets and Plumes. Proc. ASCE, J. Hyd. Div., 99, 1416-1474.

List, E.J. (1982) Turbulent Jets and Plumes. Annual Reviews in Fluid Mechanics, 114,189-

Lumley, J.L. (1978) Computational Modeling of Turbulent Flows. Advances in Applied Mechanics, V. 18, pp. 123-176. Lumley, J.L. (1971) Explanation of Thermal Plume Growth Rates. Physics Fluids, 14,
2537-2538. 
Morton, B.R. (1959) Forced Plumes. J. Fluid Mech.,2, 151-163.

Morton, B.R., Taylor, G.I. and Turner, J.S. (1956) Turbulent Gravitational Correction from Maintained and Instantaneous Sources. Proc. Roy. Soc. A234, 1-23.

Nakagome, H. and Hirata, M. (1977) The Structure of Turbulent Diffusion in an Axisymmetrical Thermal Plume. Heat Trans. \& Turbulent Buoyant Convection, (Spalding and Afgan, eds.) McGraw-Hill, NY. 367-372.

Papanicolaou, P.N. and E.J. List (1988) Investigations of Round Vertical Turbulent Buoyant Jets. J. Fluid Mech., 195, 341-391.

Papanicolaou, P.N. and E.J. List (1987) Statistical and Spectral Properties of tracer Concentration in Round Buoyant Jets. Int. J. Heat Mass Transfer, 30, 2059-2071.

Papantoniou, D. and E.J. List (1989) Large Scale Structure in the Far Field Buoyant Jets. J. Fluid Mech., 209,151-190.

Ogino, F., H. Takeuchi, I. Kudo and T. Mizushina (1980) Heated Jets Discharged Vertically into Ambients of Uniform and Linear Temperatures Profiles. Int. J. Heat Mass Transfer, $23,1581-1588$.

Rodi, W. (1986) Vertical Turbulent Buoyant Jets: Experimental Findings and Prediction Methods. Proc. Int. Symposium on Buoyant Flows, Athens, Greece, Sept. 1-5.

Rouse, J., Yih, C.S. and Humphrey, H.W. (1952) Gravitational Convection from a Boundary Source. Tellus, 4, 201-10.

Schmidt, W. (1941) Turbulent Propagation of a Stream of Air. ZAMM, V21 6.

Shabbir, A. and W.K. George (1985) Measurements of Temperature and Velocity in an Axisymmetric Turbulent Buoyant Plume. Bulletin of APS, 30, 10, Abstract DD6.

Shabbir, A. (1987) An Experimental Study of Axisymmetric Turbulent Buoyant Plume and Investigation of Closure Hypotheses. Ph. D. dissertation, Buffalo, NY.

Shabbir, A. and W.K. George (1987) An Experimental Study of an Axisymmetric Turbulent Buoyant Plume in a Neutral Environment. Proc. of 6 th Turbulent Shear Flow Conference, Tolouse, 9-3-1 to 9-3-6

Shabbir, A. Beuther, P.B. and W.K. George (1990) X-wire Response in Turbulent Flows of High Intensity Turbulence and Low Mean Velocities. Submitted to Expt. Thermal Fluid Science.

Tennekes, H. and Lumley, J.L. (1972) A First Course in Turbulence, MIT Press.

Townsend, A.A. (1956) Structure of Turbulent Shear Flows, Cambridge University Press.

Tutu, N.K. and Chevray, R. (1975) Cross-wire Anemometry in High Intensity Turbulence, J. Fluid Mech., 71,785 . 
Zel'dovich, Ya B. (1937) Limiting Laws for Turbulent Flows in Free Convection, Zh. EKSP. Theoret. Fiz 7(12), 1463. 
TABLE I - SOURCE CONDITIONS

\begin{tabular}{|l|c|c|c|c|c|}
\hline Experiment & $T_{o}($ Celcius $)$ & $U_{o}(\mathrm{~m} / \mathrm{s})$ & $F_{o}\left(\mathrm{~m}^{4} / \mathrm{s}^{3}\right)$ & $M_{\circ}\left(\mathrm{m}^{4} / \mathrm{s}^{2}\right)$ & $R_{o}$ \\
\hline Two wire probe & 295 & 0.98 & 0.0127 & 0.0030 & 0.745 \\
\hline Three wire probe & 295 & 0.86 & 0.0115 & 0.0023 & 0.585 \\
\hline
\end{tabular}

TABLE II

\begin{tabular}{|l|c|c|c|c|c|c|c|c|}
\hline Reference & $A_{t}$ & $A_{u}$ & $B_{t}$ & $B_{u}$ & $Y_{.5 t}$ & $Y_{.5 u}$ & $t / \Delta T_{C L}$ & $\dot{u} / U_{C L}$ \\
\hline Rouse et al. & 11.0 & 4.7 & 71 & 96 & 0.095 & 0.084 & - & - \\
\hline George et al. & 9.1 & 3.4 & 65 & 55 & 0.104 & 0.112 & 0.38 & 0.28 \\
\hline Nakagoma \& Hirata & 11.5 & 3.89 & 48.1 & 63 & 0.105 & 0.12 & 0.36 & 0.25 \\
\hline Papanicolaou \& List & 14.28 & 3.85 & 80 & 90 & 0.093 & 0.0877 & 0.42 & 0.25 \\
\hline Chen \& Rodi & 9.35 & 3.5 & 65 & 55 & 0.100 & 0.112 & - & - \\
\hline Present study & 9.4 & 3.4 & 68 & 60 & 0.101 & 0.1075 & 0.40 & 0.33 \\
\hline
\end{tabular}

TABLE III

\begin{tabular}{|l|c|c|c|c|}
\hline Experiment & $\xi=z / L$ & $\frac{2 \pi}{M_{0}} \int_{0}^{\infty}\left(W^{2}+w^{2}-u^{2}\right) r d r$ & $\frac{d}{d \xi}\left\{\frac{2 \pi}{M_{0}} \int_{0}^{\infty}\left(W^{2}+w^{2}-u^{2}\right) r d r\right\}$ & $2 \pi \frac{L}{M_{0}} \int_{0}^{\infty}(g \beta \Delta T) r d r$ \\
\hline \multirow{3}{*}{$\begin{array}{l}\text { Two wire } \\
\text { probe }\end{array}$} & 6.59 & 3.42 & 0.858 & 0.854 \\
\cline { 2 - 5 } & 7.65 & 4.67 & 0.983 & 0.898 \\
\cline { 2 - 5 } & 9.363 & 6.136 & 1.036 & 0.961 \\
\hline \multirow{3}{*}{$\begin{array}{l}\text { Three wire } \\
\text { probe }\end{array}$} & 6.84 & 4.867 & 0.923 & 0.865 \\
\cline { 2 - 5 } & 9.09 & 7.17 & 0.928 & 0.951 \\
\cline { 2 - 5 } & 11.23 & 10.14 & 1.031 & 1.021 \\
\cline { 2 - 5 } & 13.17 & 10.53 & 1.036 & 1.076 \\
\cline { 2 - 5 } & 15.21 & 13.13 & 1.087 & 1.129 \\
\hline
\end{tabular}




\section{FIGURE CAPTIONS}

Figure 1. Nomenclature.

Figure 2. Sketch of the facility.

Figure 3. Asymptotic plume behavior corresponding to the source conditions of Table I.

Figure 4. Change in ambient temperature as a function of height and time for one of the

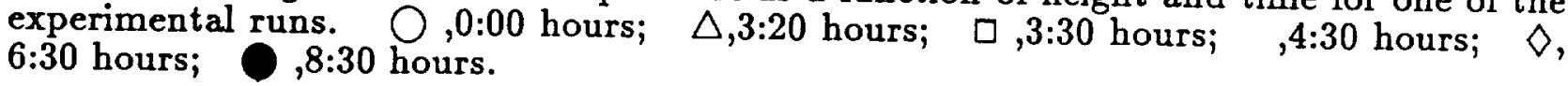

Figure 5. Mean buoyancy profile measured with a two wire probe. Close symbols refer to two wire probe measurements and open symbols refer to the three wire probe measurements. $\mathrm{z} / \mathrm{D}=11.81 ; \quad \mathrm{z} / \mathrm{D}=13.7 ; \quad \mathrm{A}, \mathrm{z} / \mathrm{D}=16.77 ; \quad \mathrm{z} / \mathrm{D}=19.5 \quad \mathrm{Q}, \mathrm{z} / \mathrm{D}=10.55$; $\mathrm{O}, \mathrm{z} / \mathrm{D}=14 ; \Delta, \mathrm{z} / \mathrm{D}=17.3 ; \quad \nabla, \mathrm{z} / \mathrm{D}=20.31 ; \diamond, \mathrm{z} / \mathrm{D}=23.46$

Figure 6. Mean buoyancy profile measured with thermocouples. $\quad \square, z / D=10$; $\mathrm{z} / \mathrm{D}=14 ; \triangle, \mathrm{z} / \mathrm{D}=19 ; \quad+\mathrm{z} / \mathrm{D}=25$.

Figure 7. Mean vertical and radial velocity profiles. Figure captions same as in figure 5.

Figure 8. Profile of the temperature fluctuations. Figure captions same as in figure 5.

Figure 9. Profile of the vertical turbulent heat flux. Figure captions same as in figure 5.

Figure 10. Profile of the radial turbulent heat flux. Figure captions same as in figure 5.

Figure 11. Profile of the vertical velocity fluctuations. Figure captions same as in figure 5.

Figure 12. Profile of the radial velocity fluctuations. Figure captions same as in figure 5.

Figure 13. Profile of the shear stress. Figure captions same as in figure 5.

Figure 14. Profiles of third moments involving temperature. Figure captions same as in figure 5 .

Figure 15. Profiles of third moments involving velocity only. Figure captions same as in figure 5 .

Figure 16. Ratio of the local buoyancy flux (obtained by integrating the measurements) to the source buoyancy flux. Figure captions same as in figure 5.

Figure 17. Normalized mean momentum flux $\frac{M}{M_{0}}$ at different heights plotted as a function of $\xi^{4 / 3}$. For symbol's meaning see caption of figure 5 .

Figure 18a. Balance of the mean energy differential equation.

Figure 18b. Sketch showing the different zones of the plume based on the mean energy 
differential equation balance.

Figure 19a. Balance of the mean momentum differential equation.

Figure 19b. Sketch showing the different zones of the plume based on the mean momentum differential equation balance.

Figure 20a. Balance for the $\overline{t^{2}} / 2$ equation.

Figure 20b. Sketch showing the different zones of the plume based on the $\overline{t^{2}} / 2$ balance.

Figure 21a. Balance of the $\overline{q^{2}} / 2$ equation.

Figure $21 \mathrm{~b}$. Sketch showing the different zones of the plume based on the $\overline{q^{2}} / 2$ balance.

Figure 22. Radial profile of turbulence Reynolds number at a height of 1 meter. 


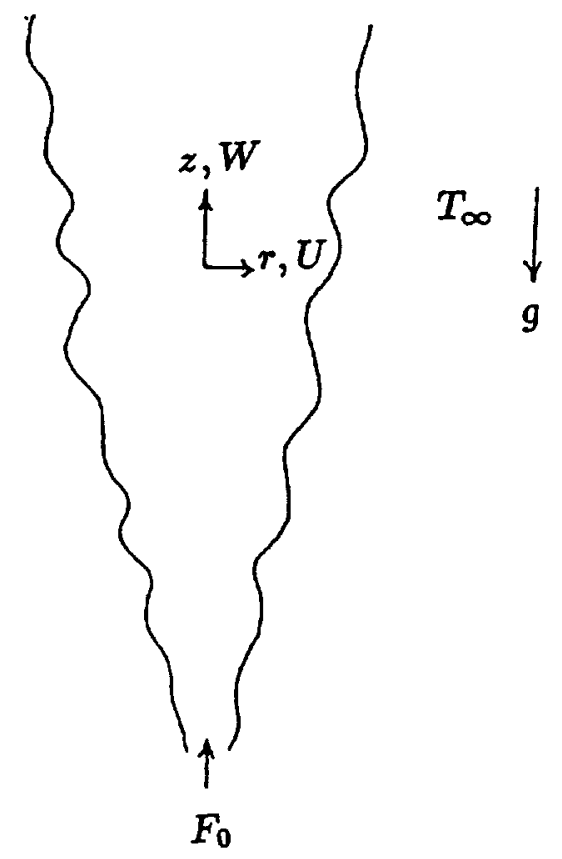

Figure 1 


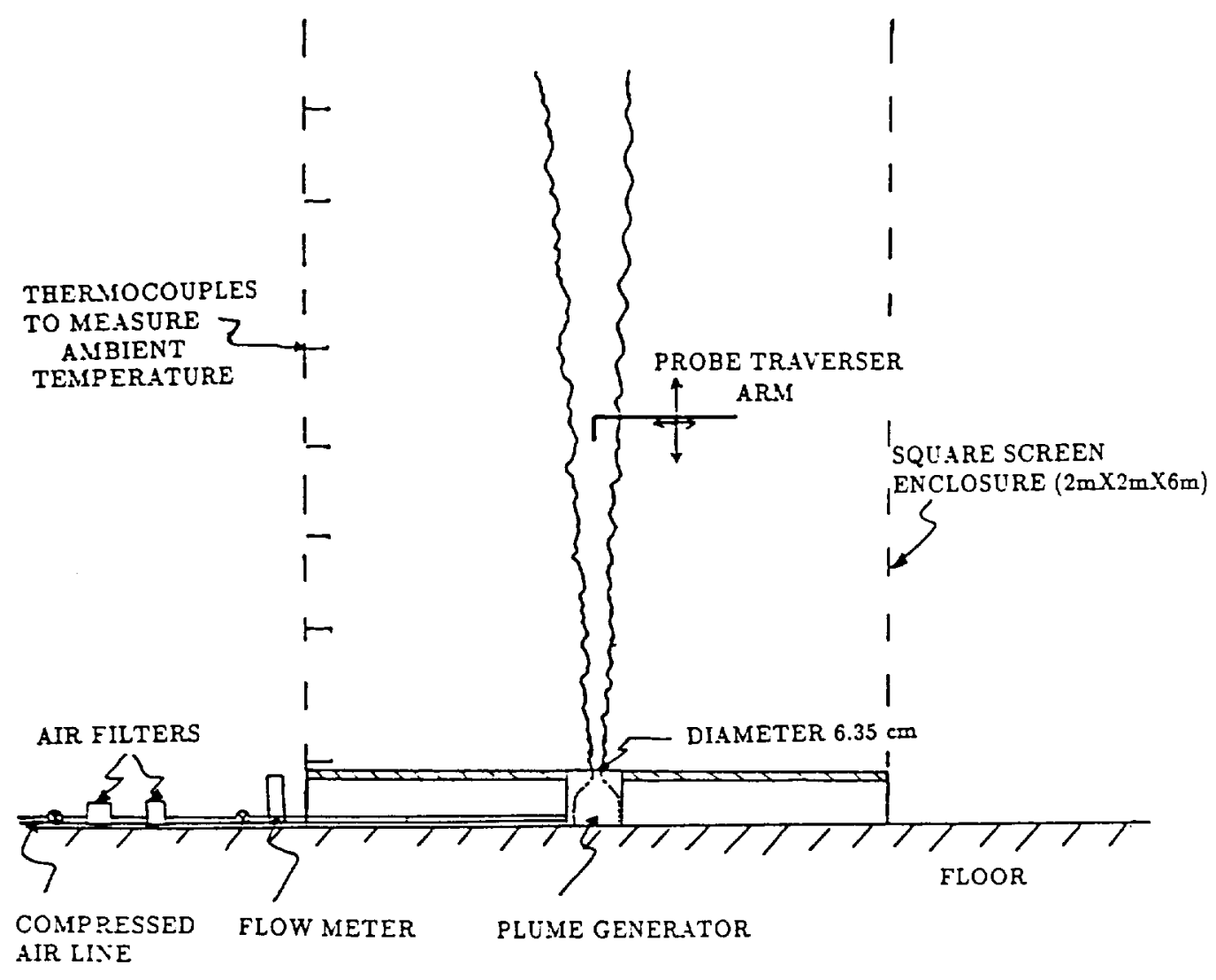

Figure 2 


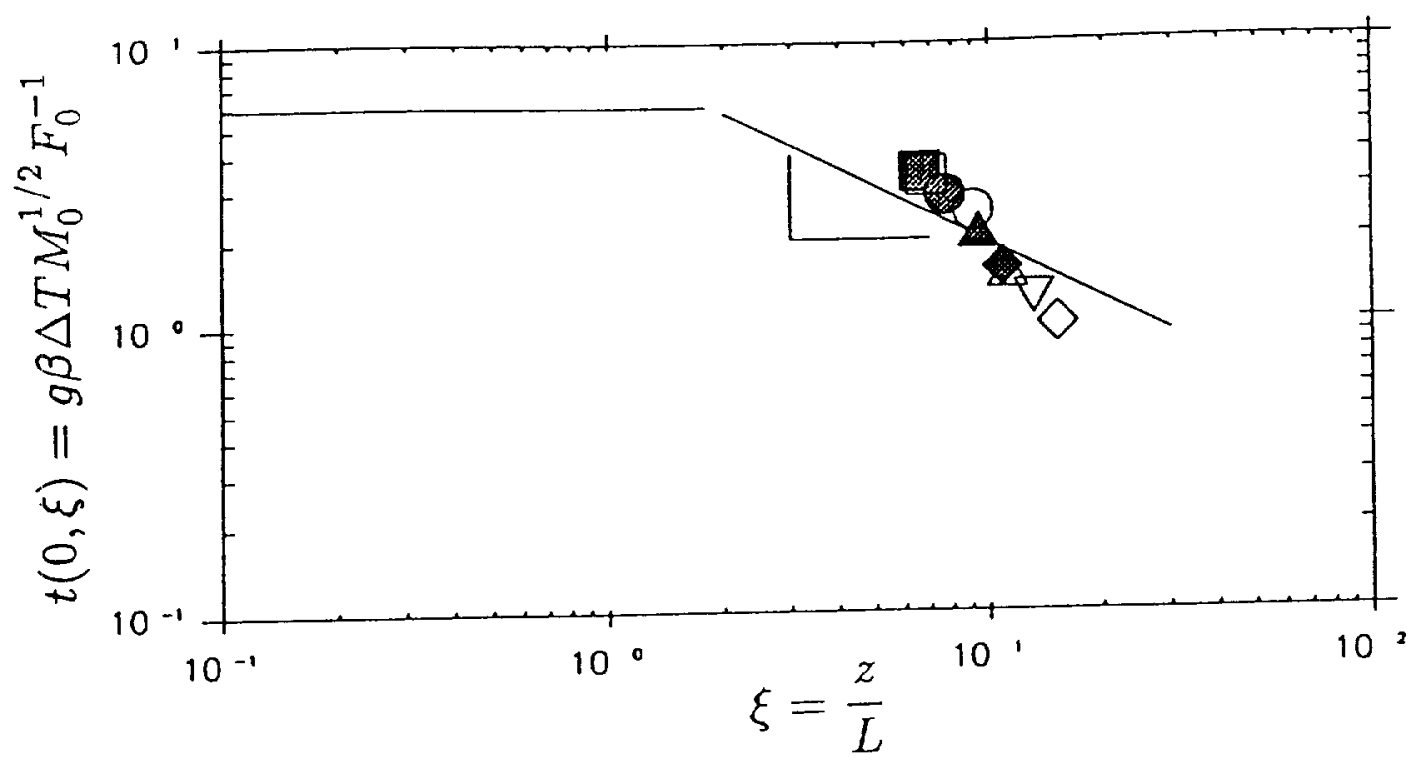

Figure 3

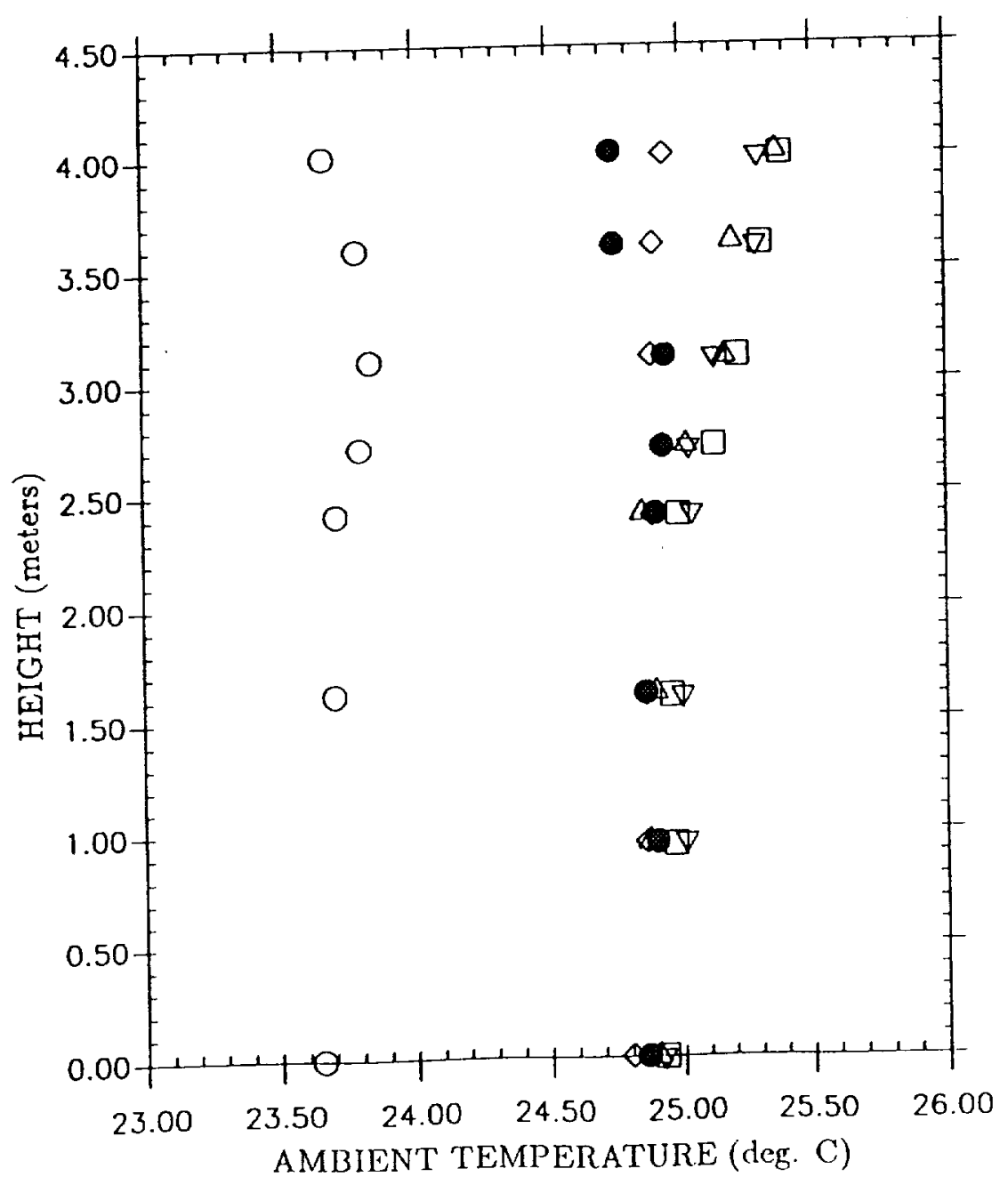

Figure 4 


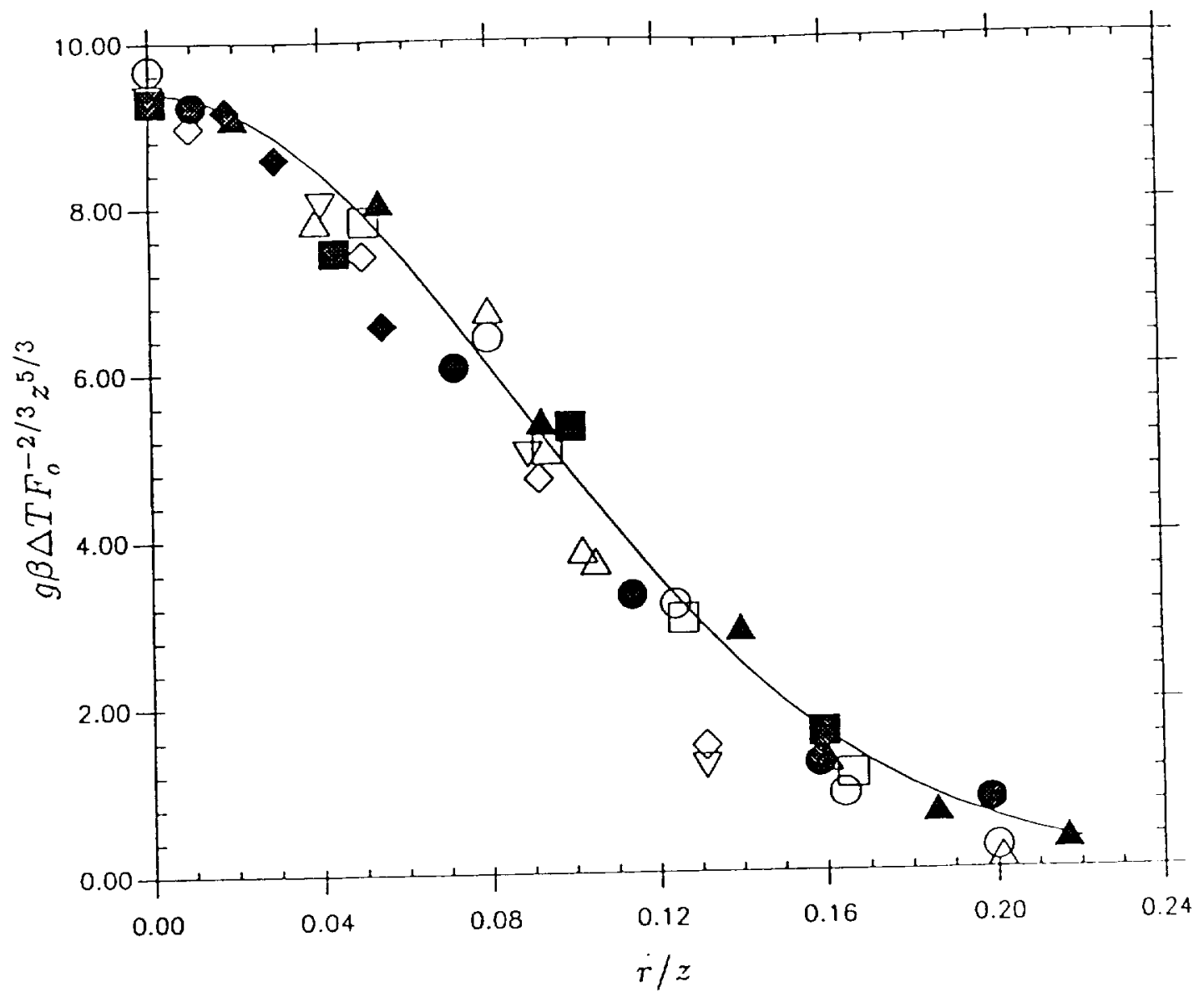

Figure 5

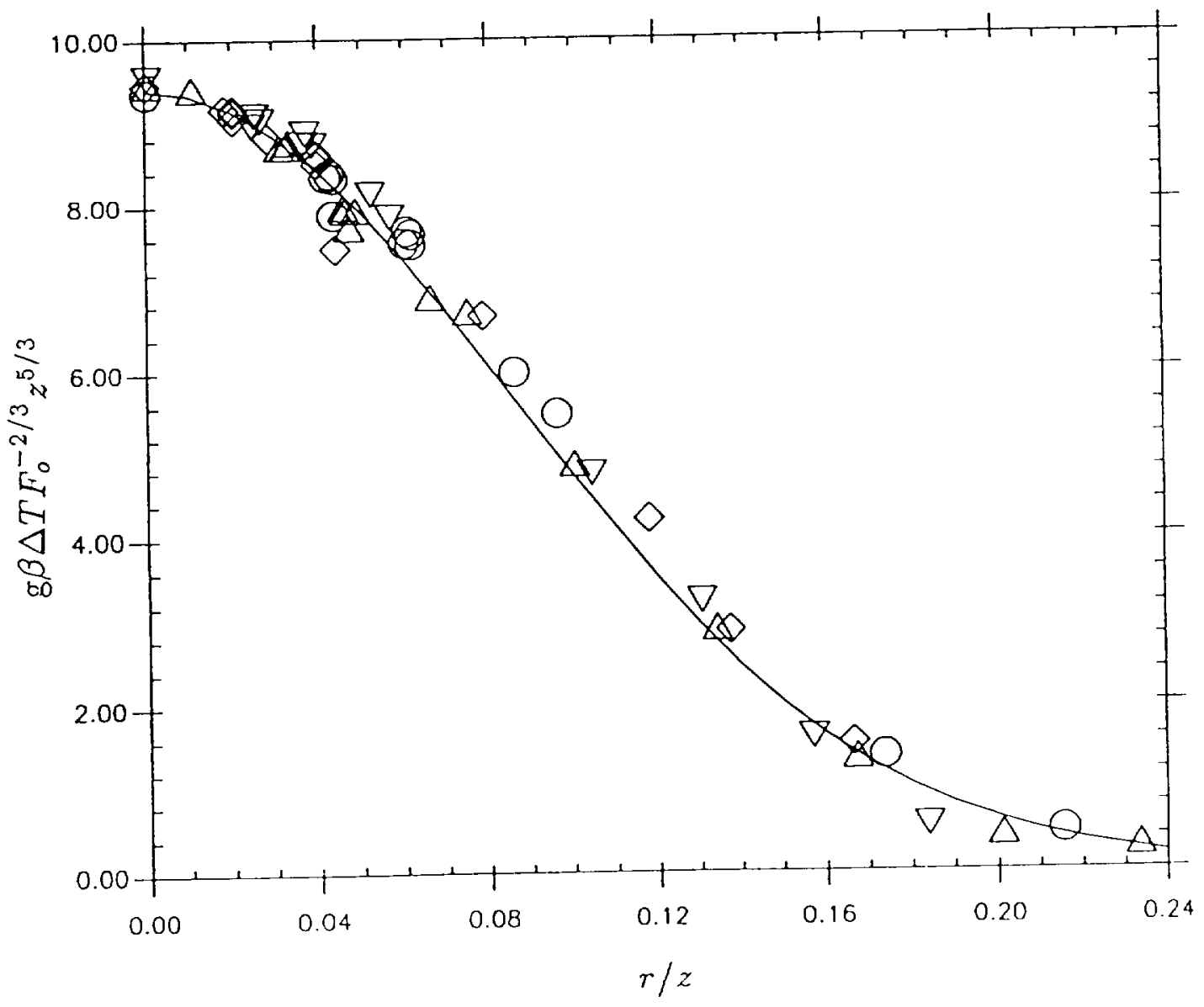

Pigure 6

41 


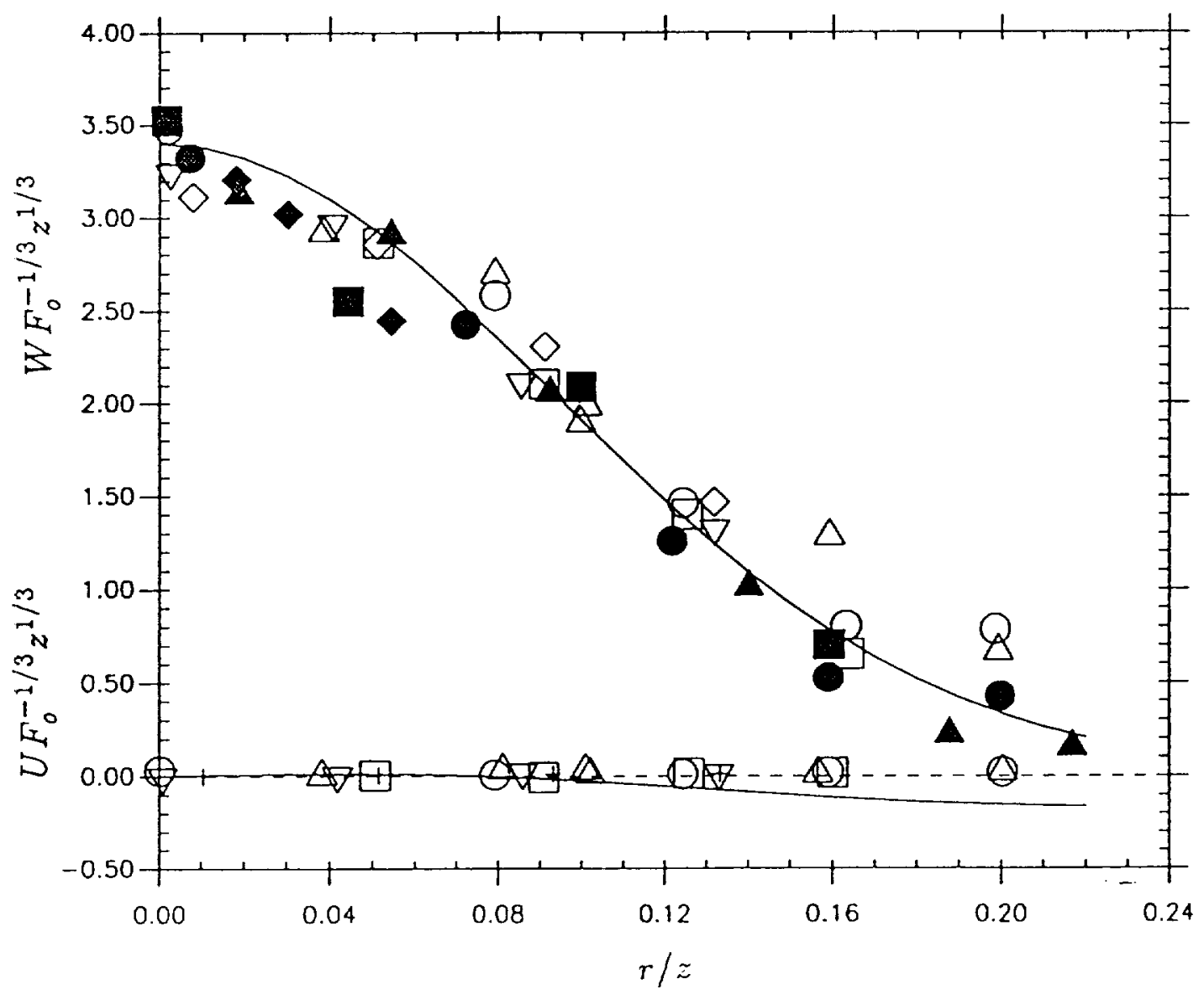

Figure 7 


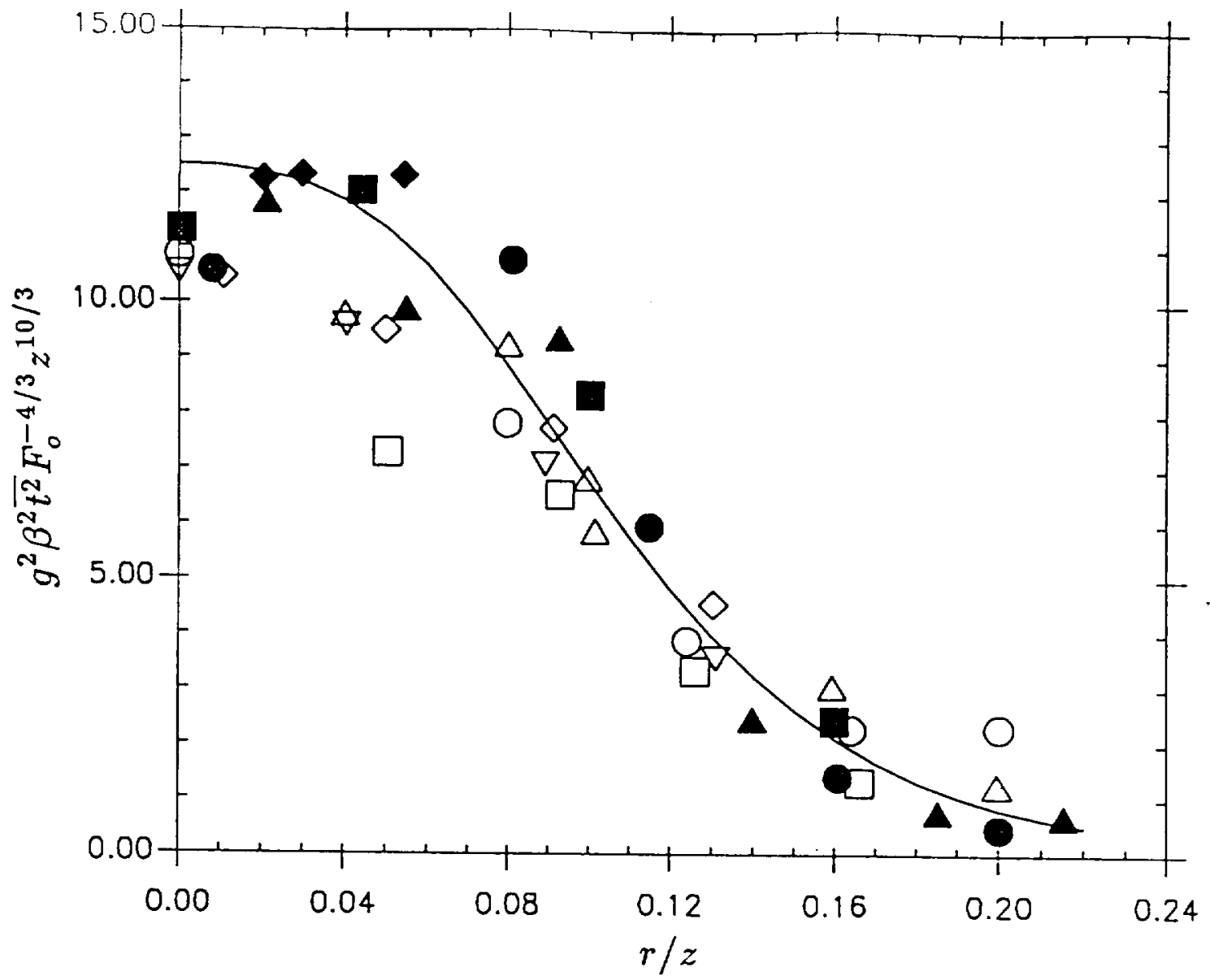

Figure 8

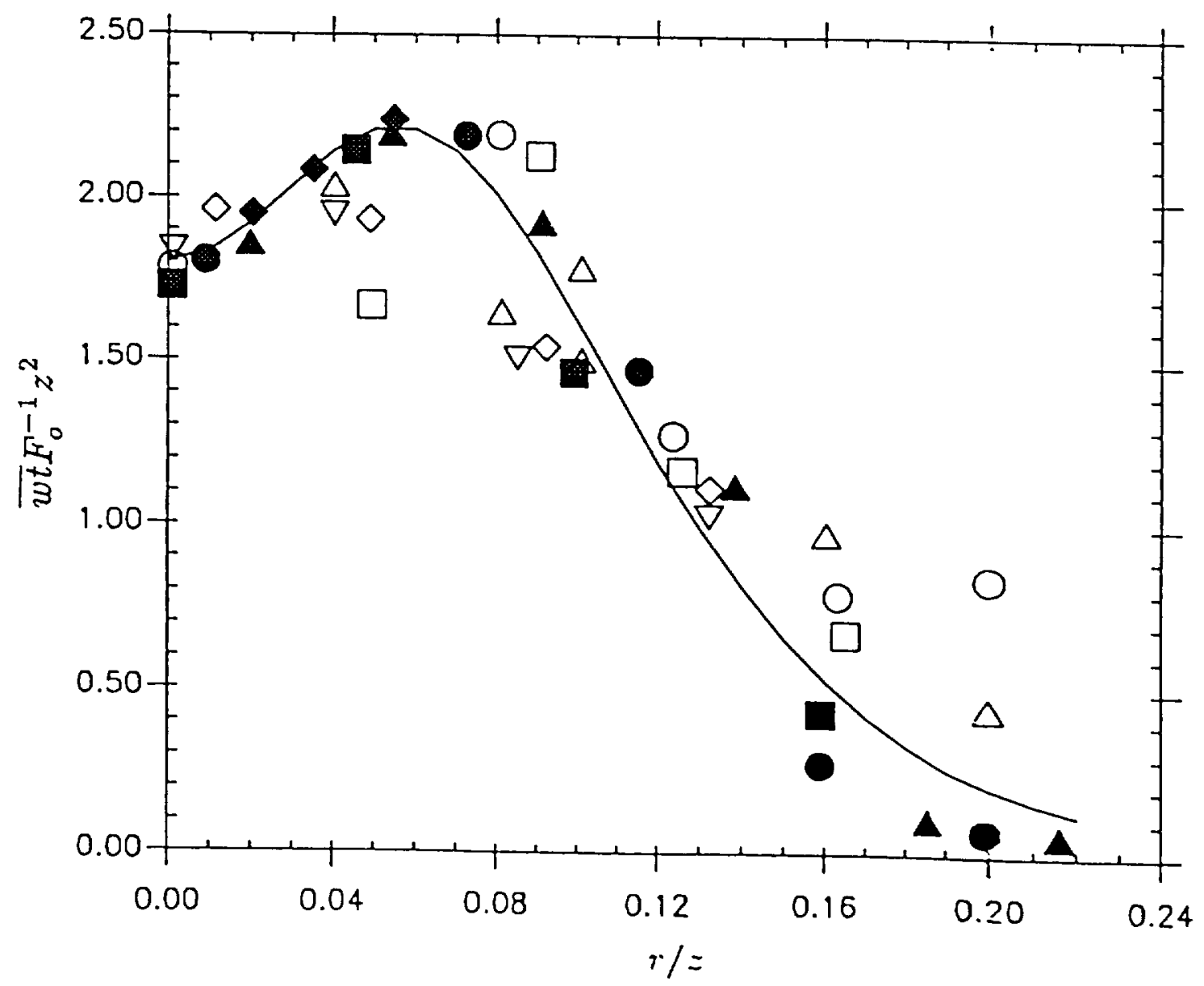

Figure 9 


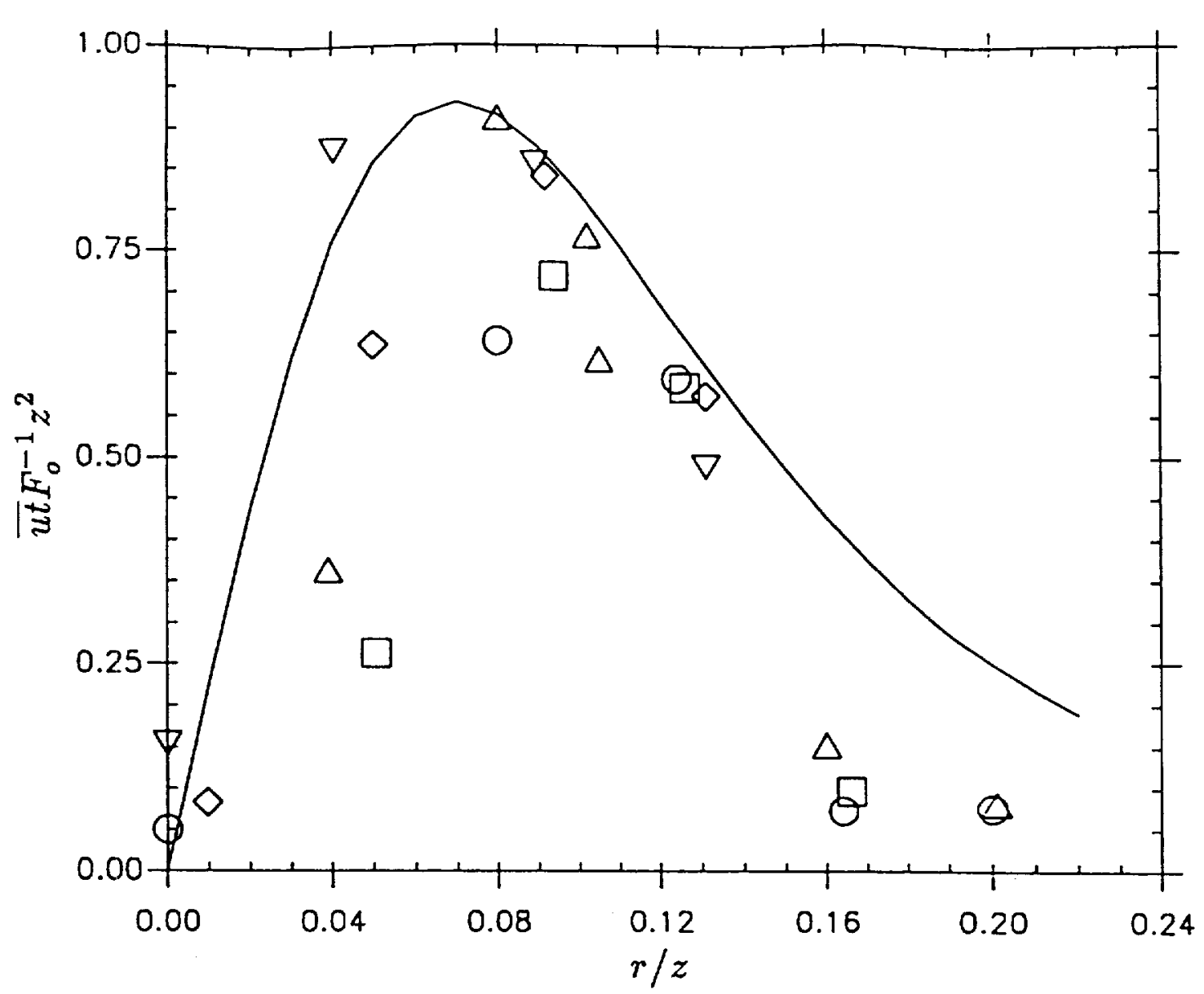

Figure 10

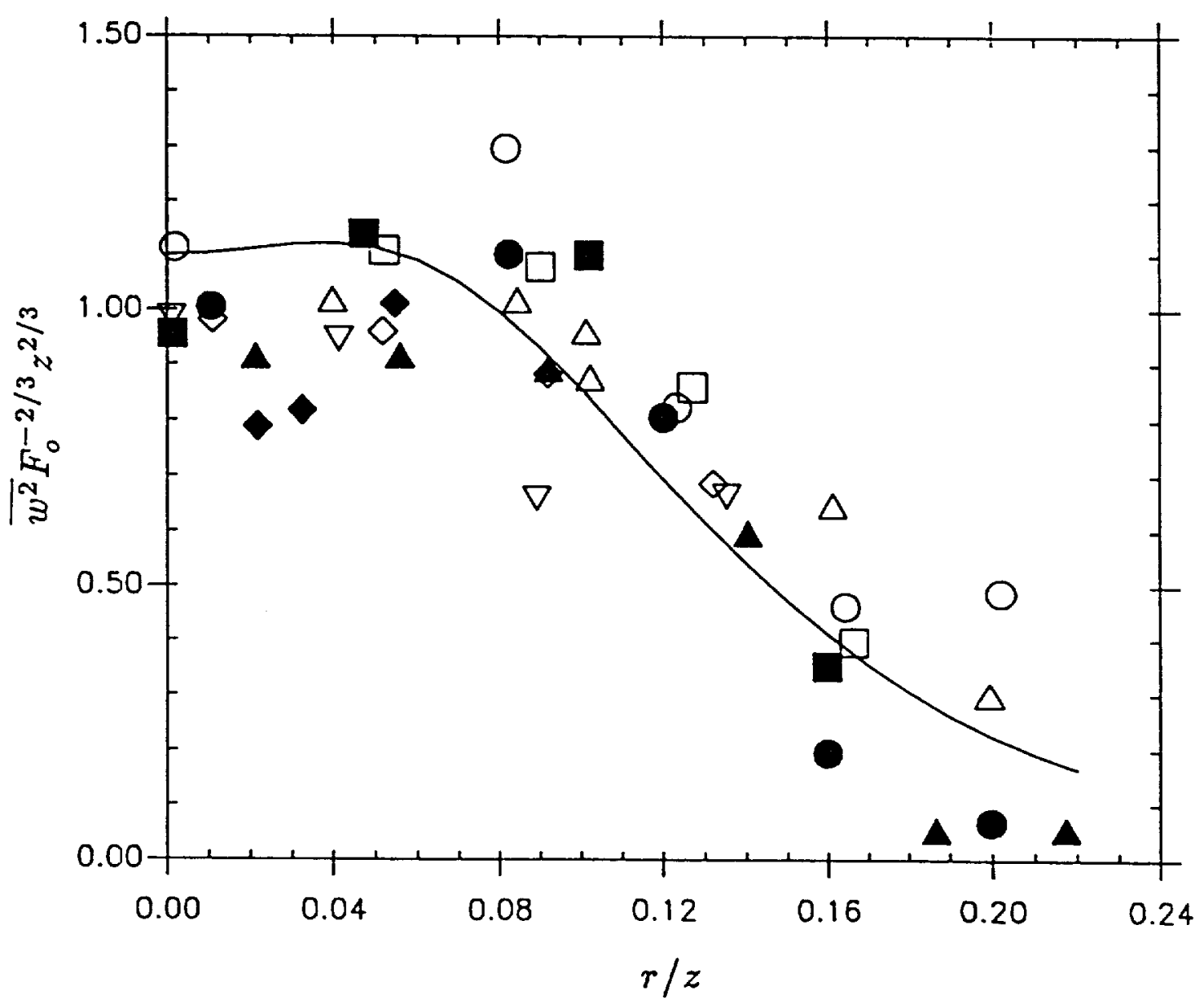

Figure 11 


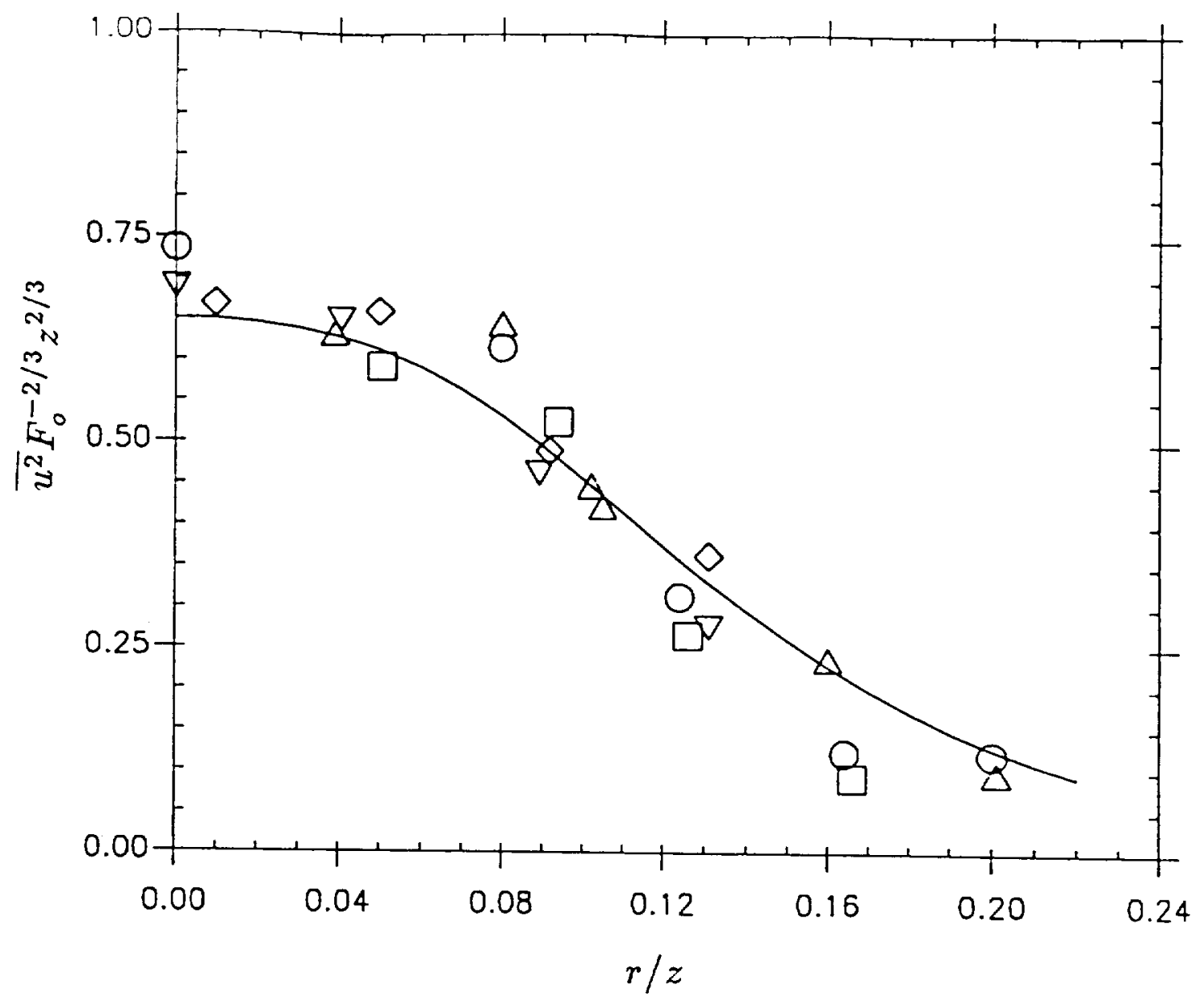

Figure 12

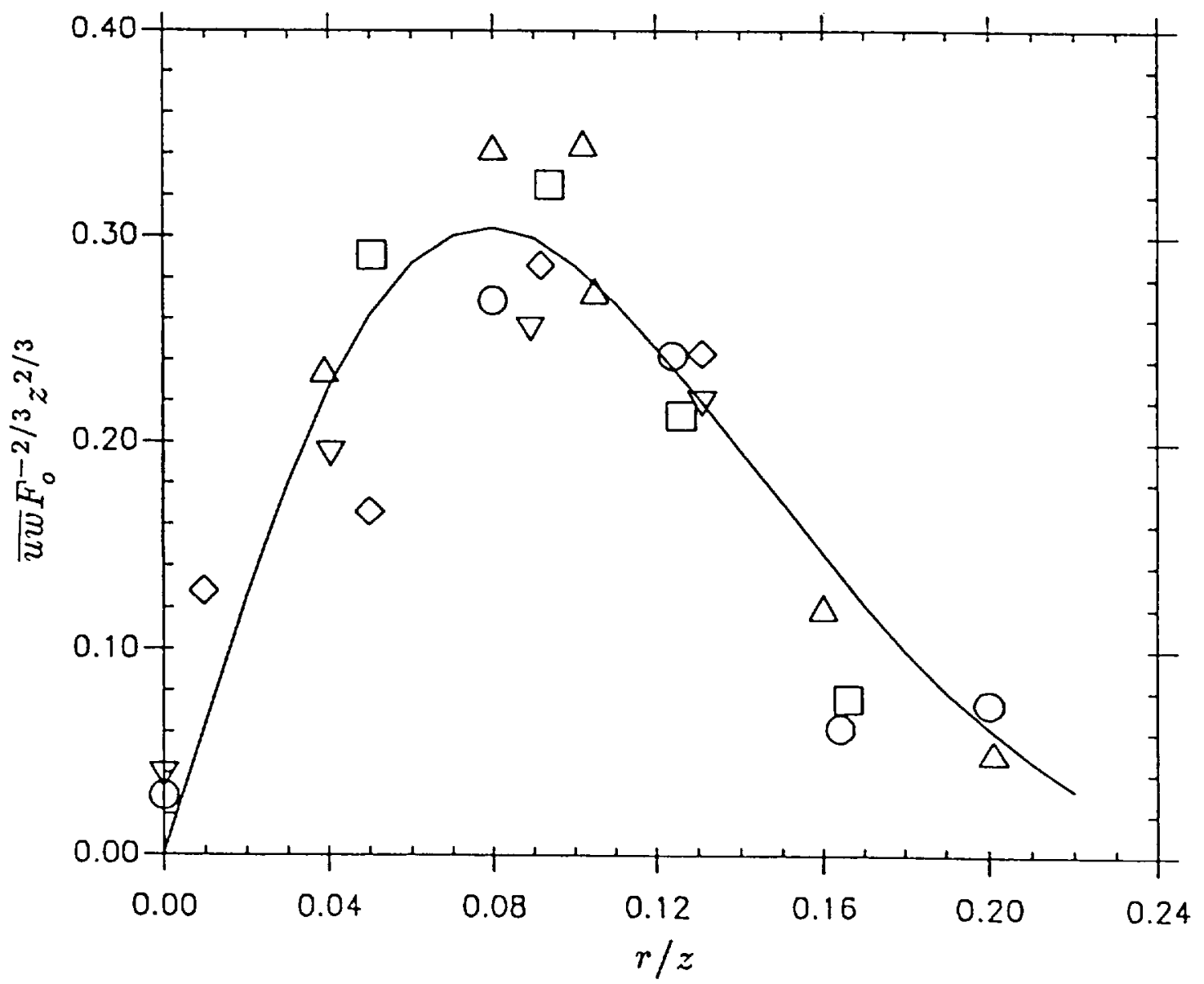

Figure 13 

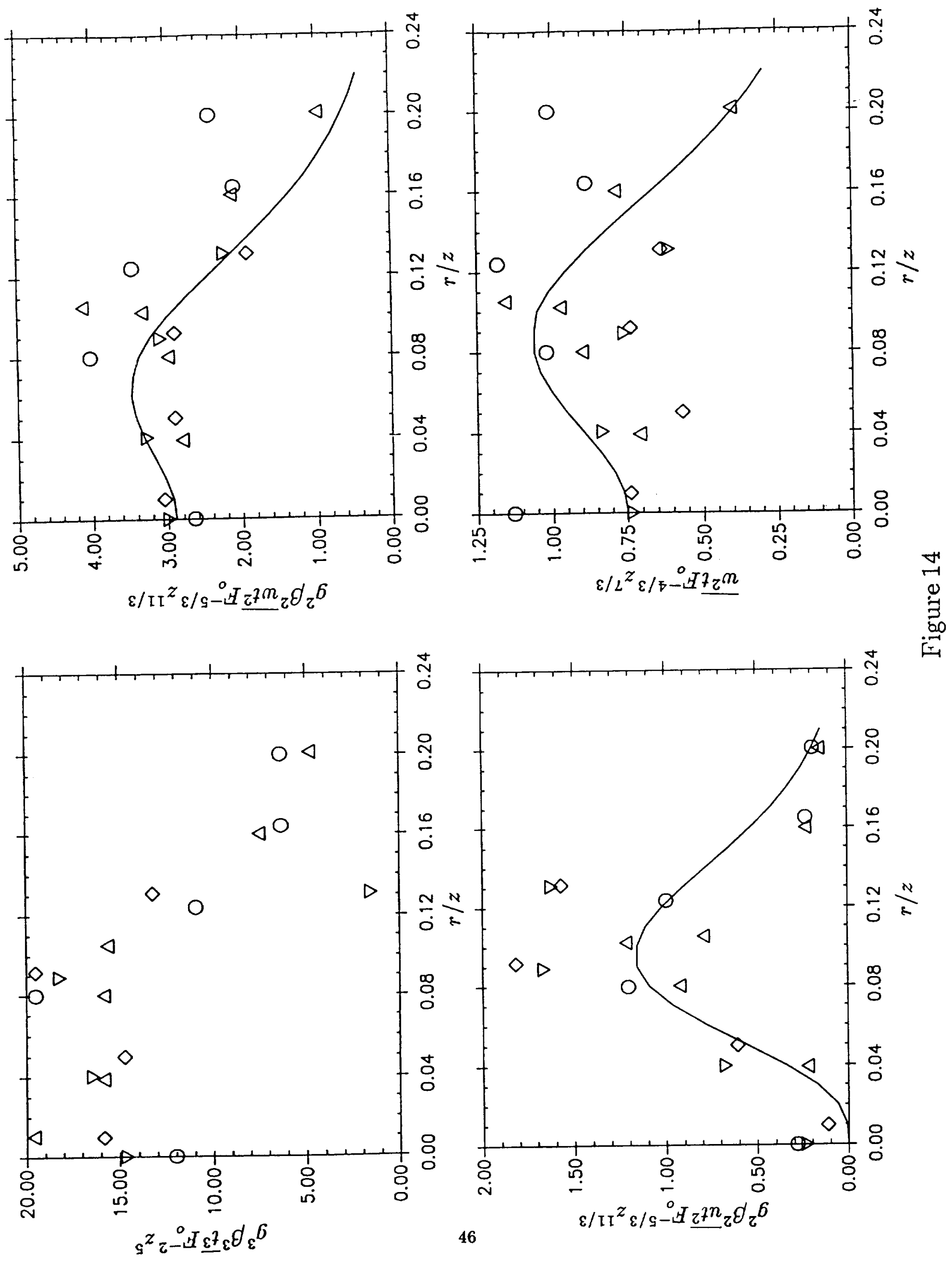


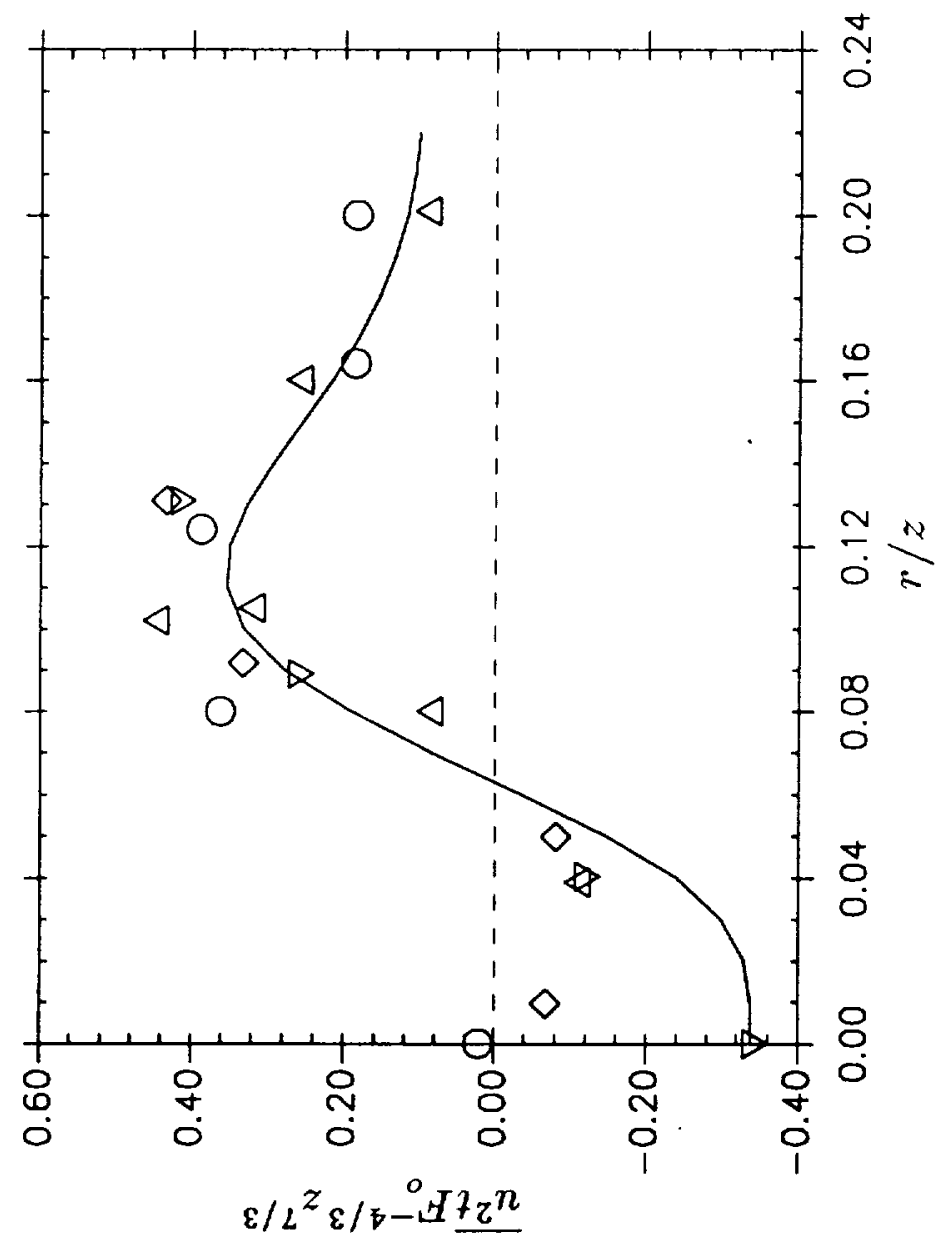

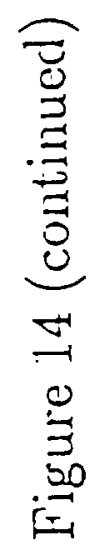



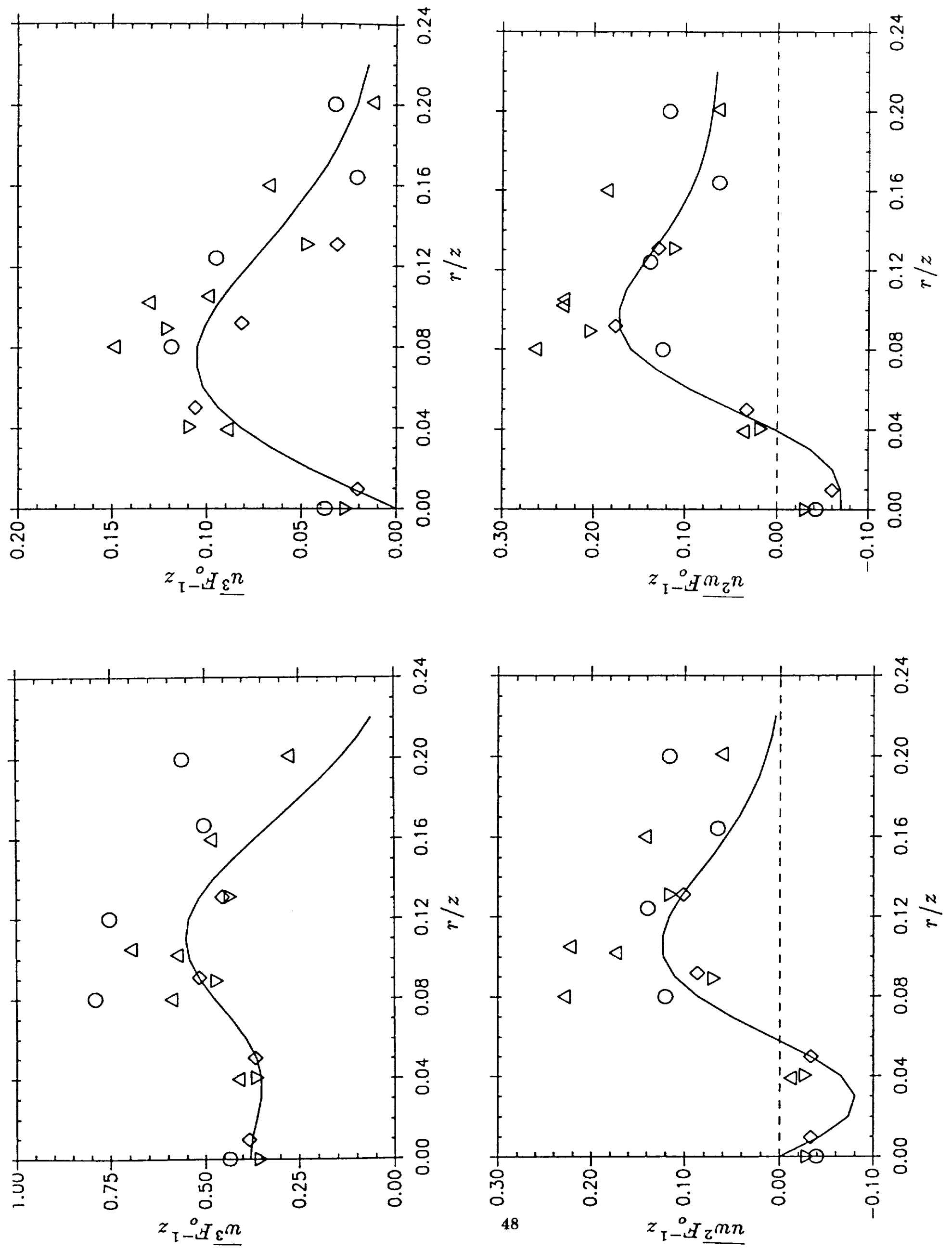


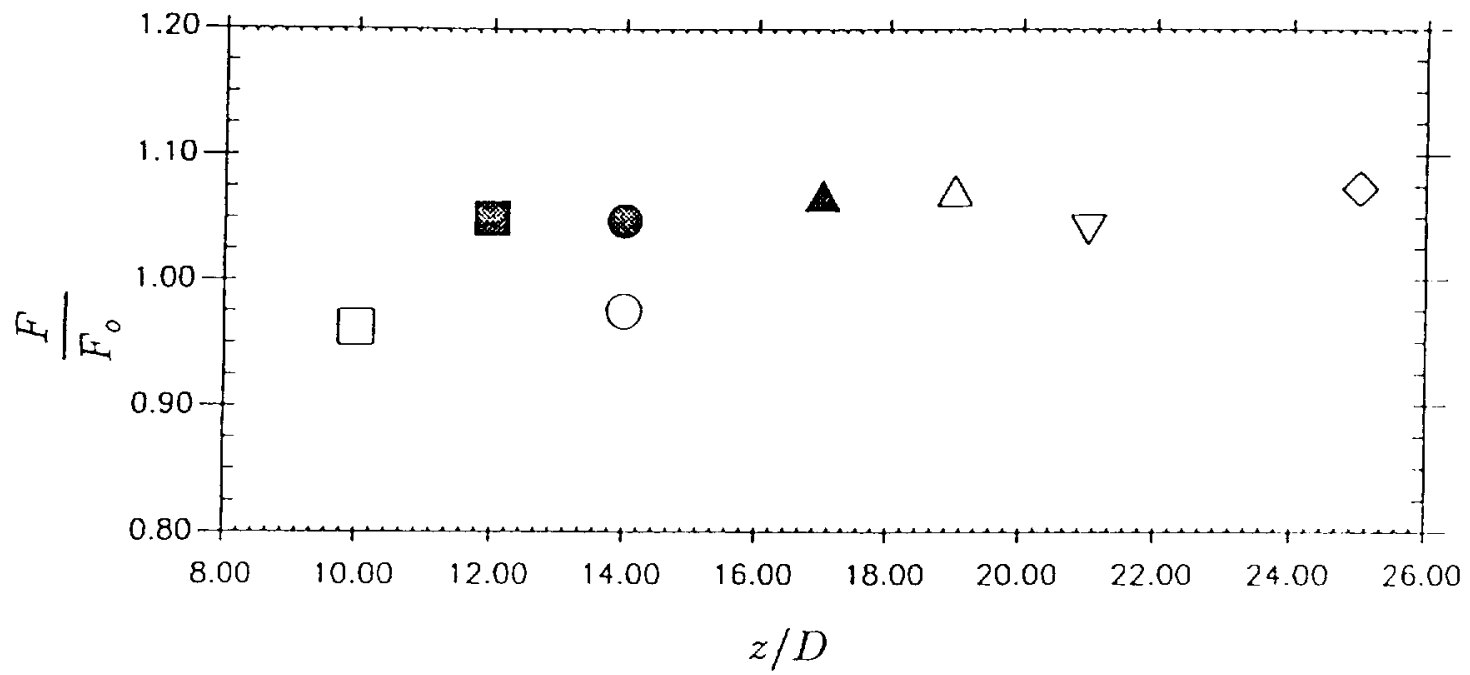

Figure 16

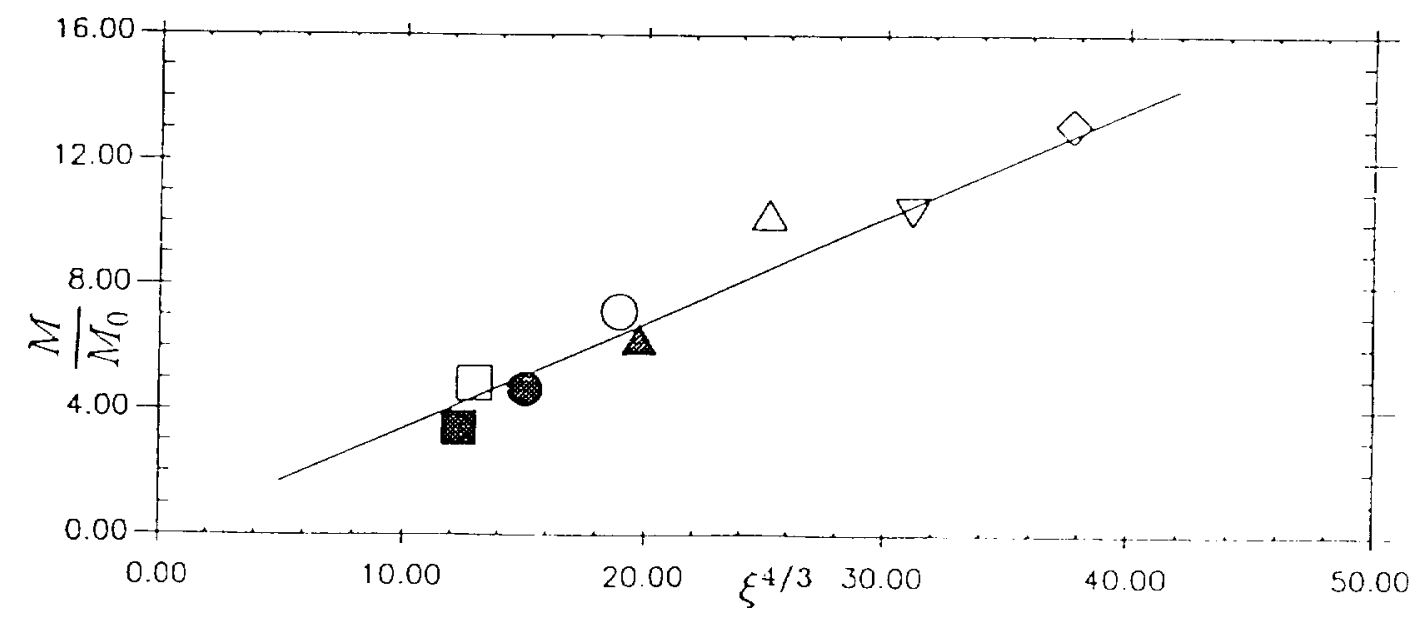

Figure 17 


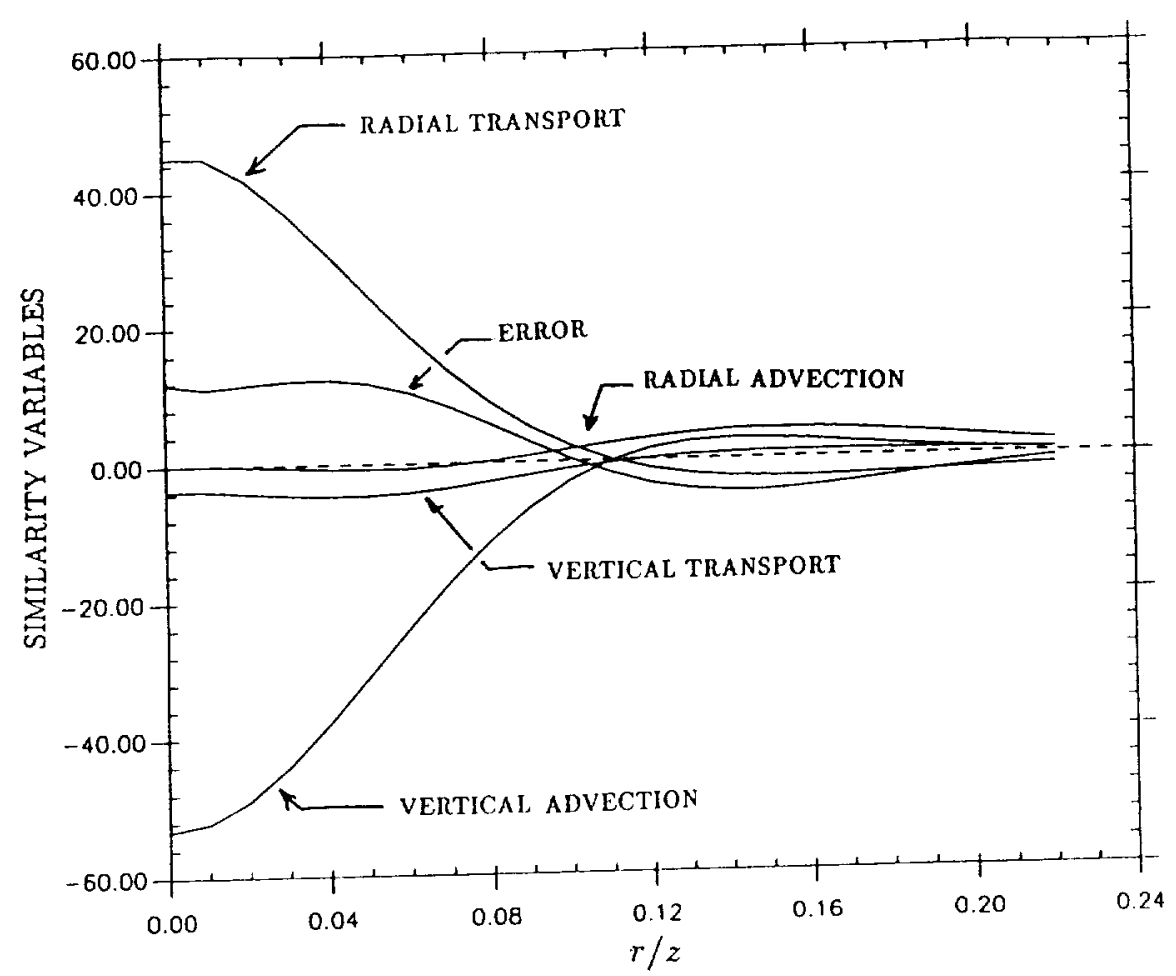

Figure 18a
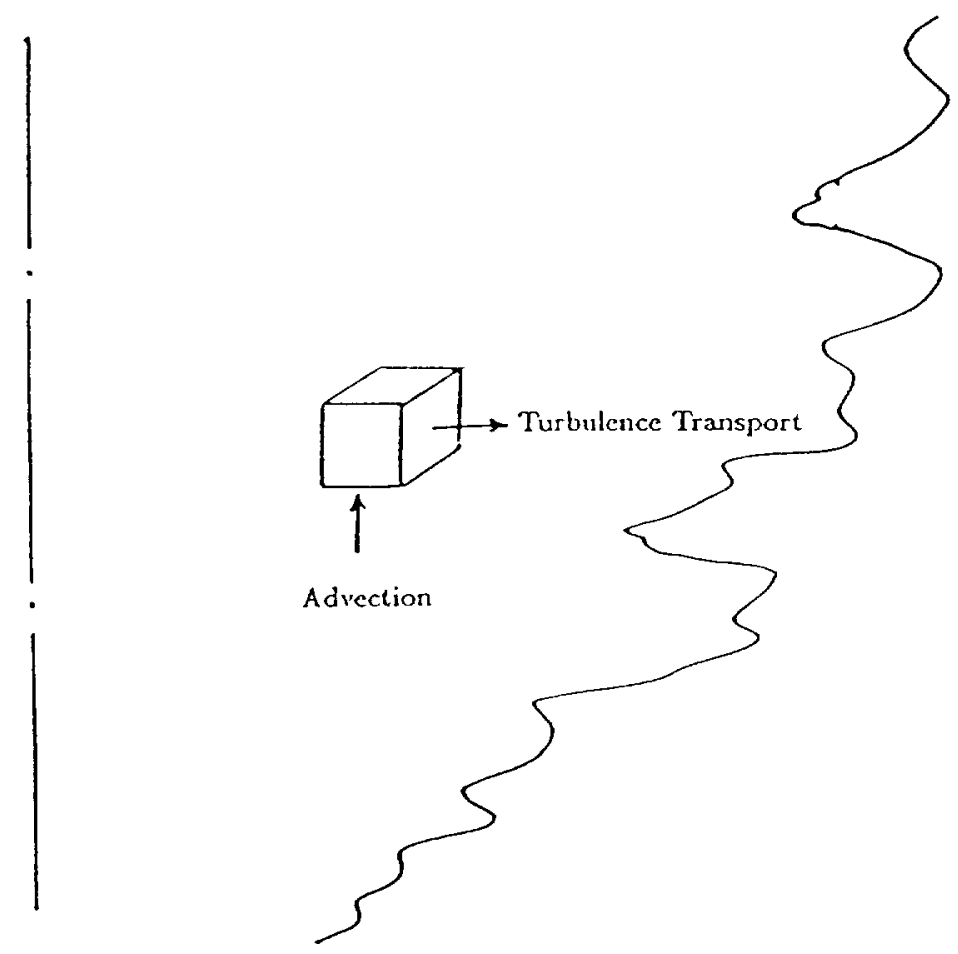

Figure 18b 


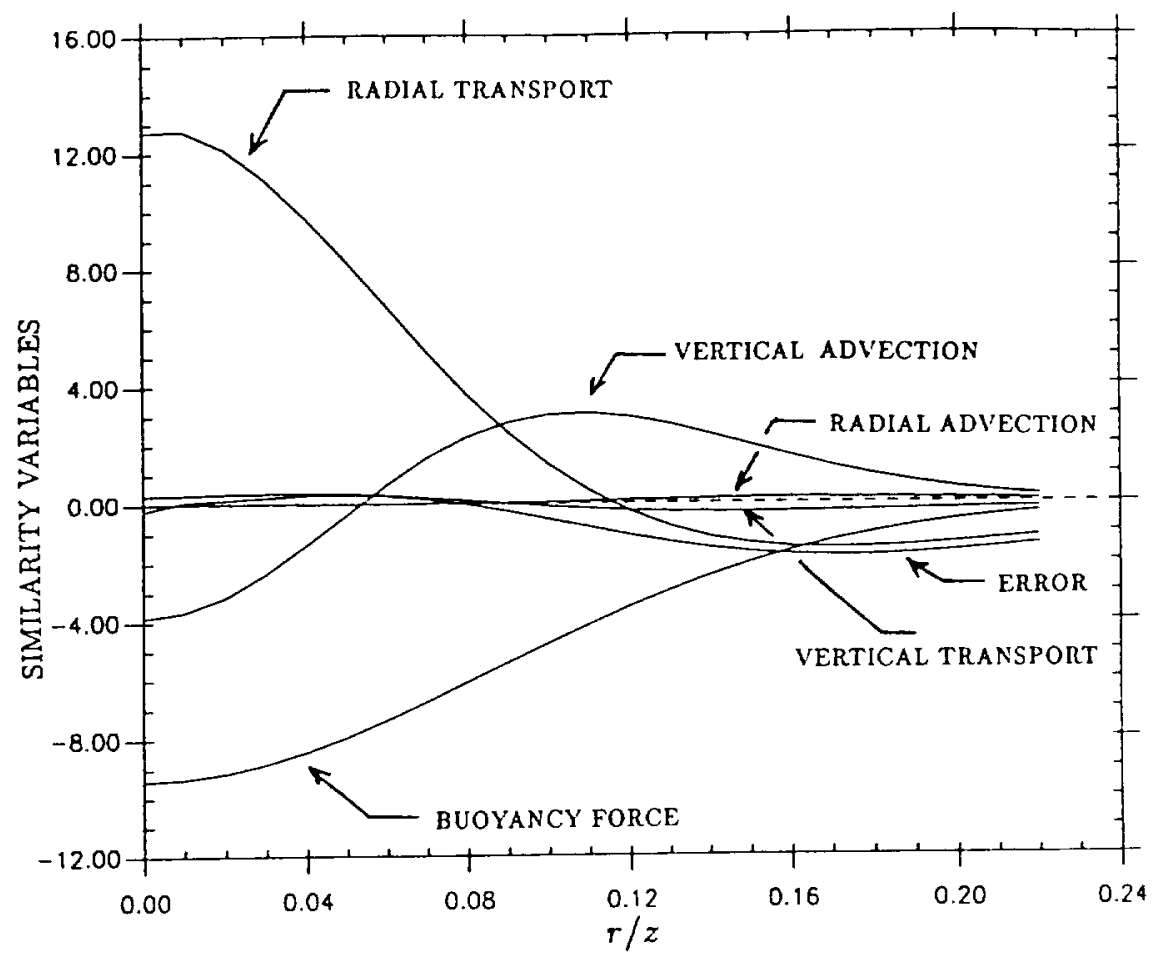

Figure 19a

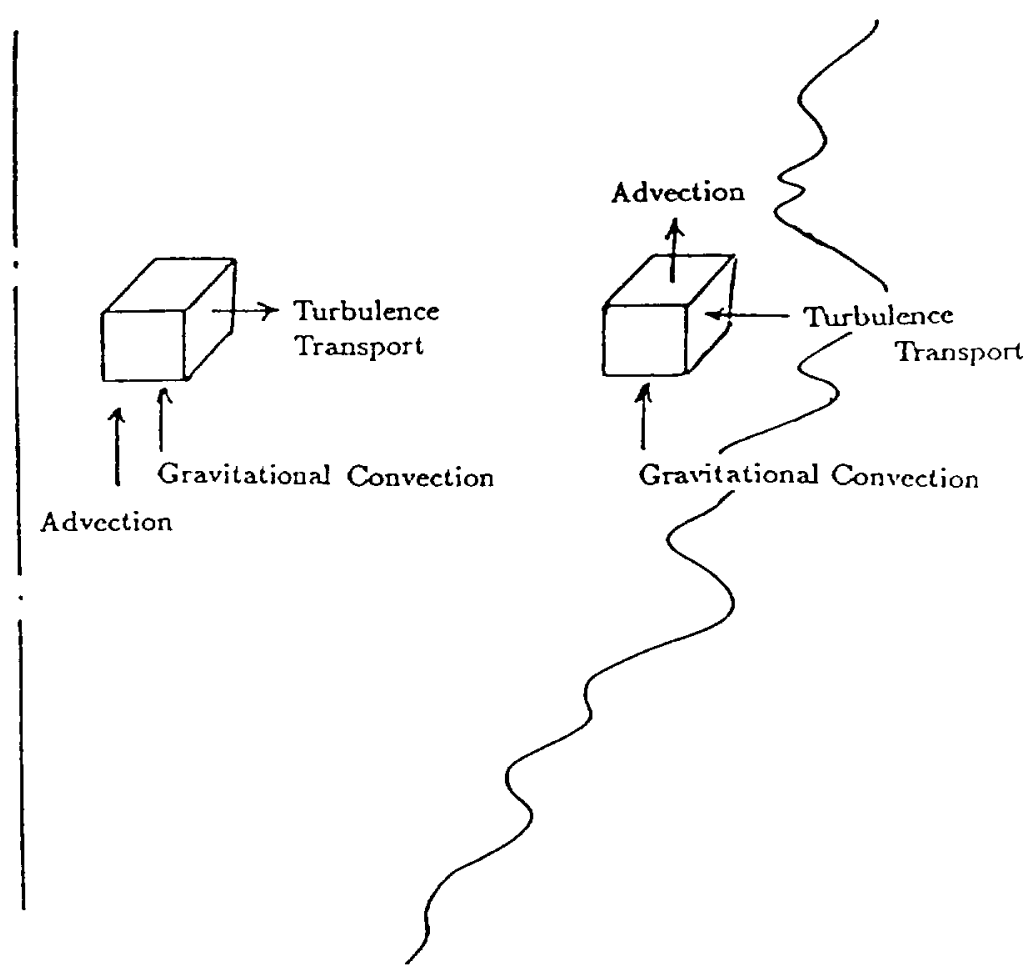

Figure 19b 


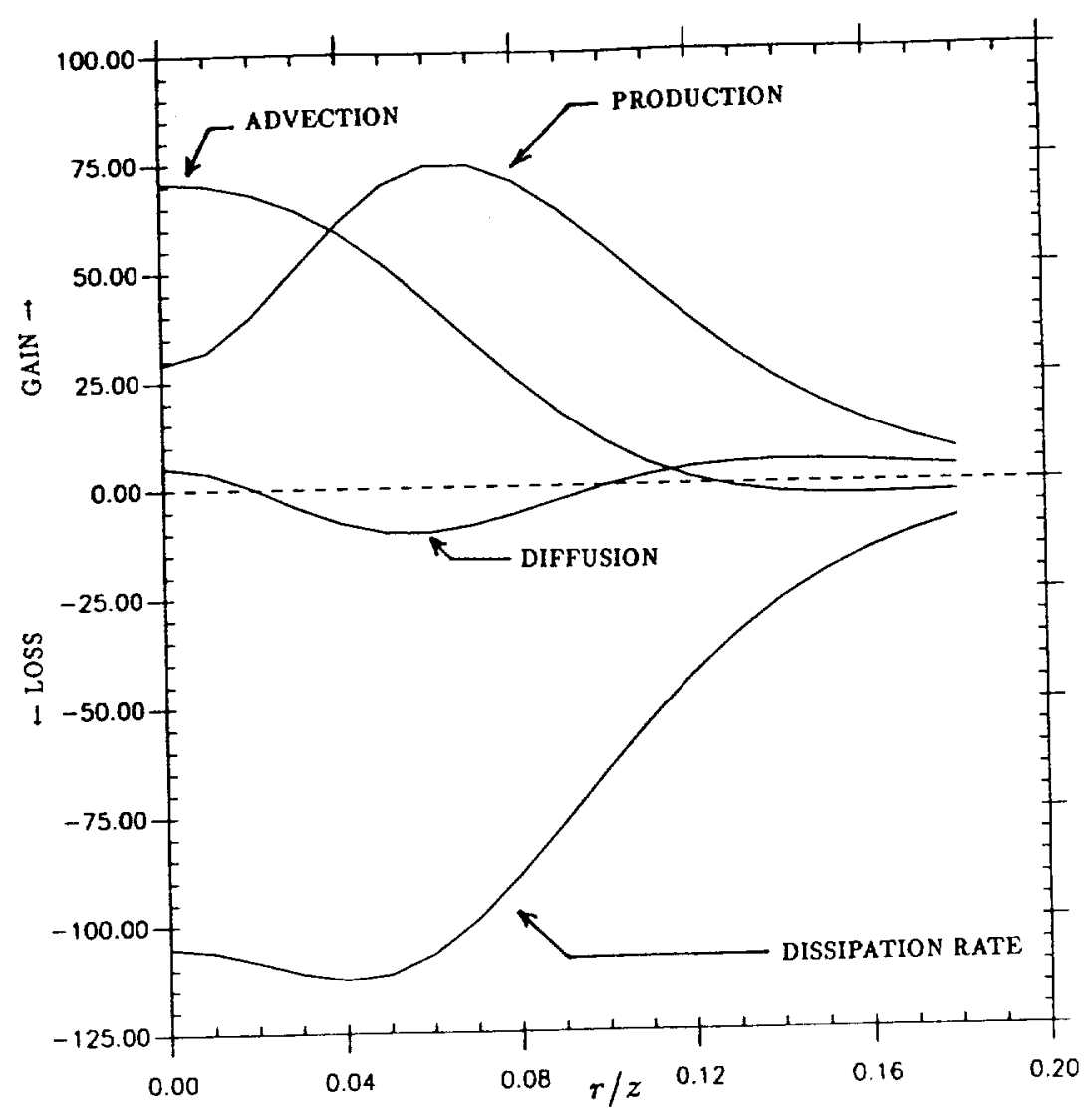

Figure 20a

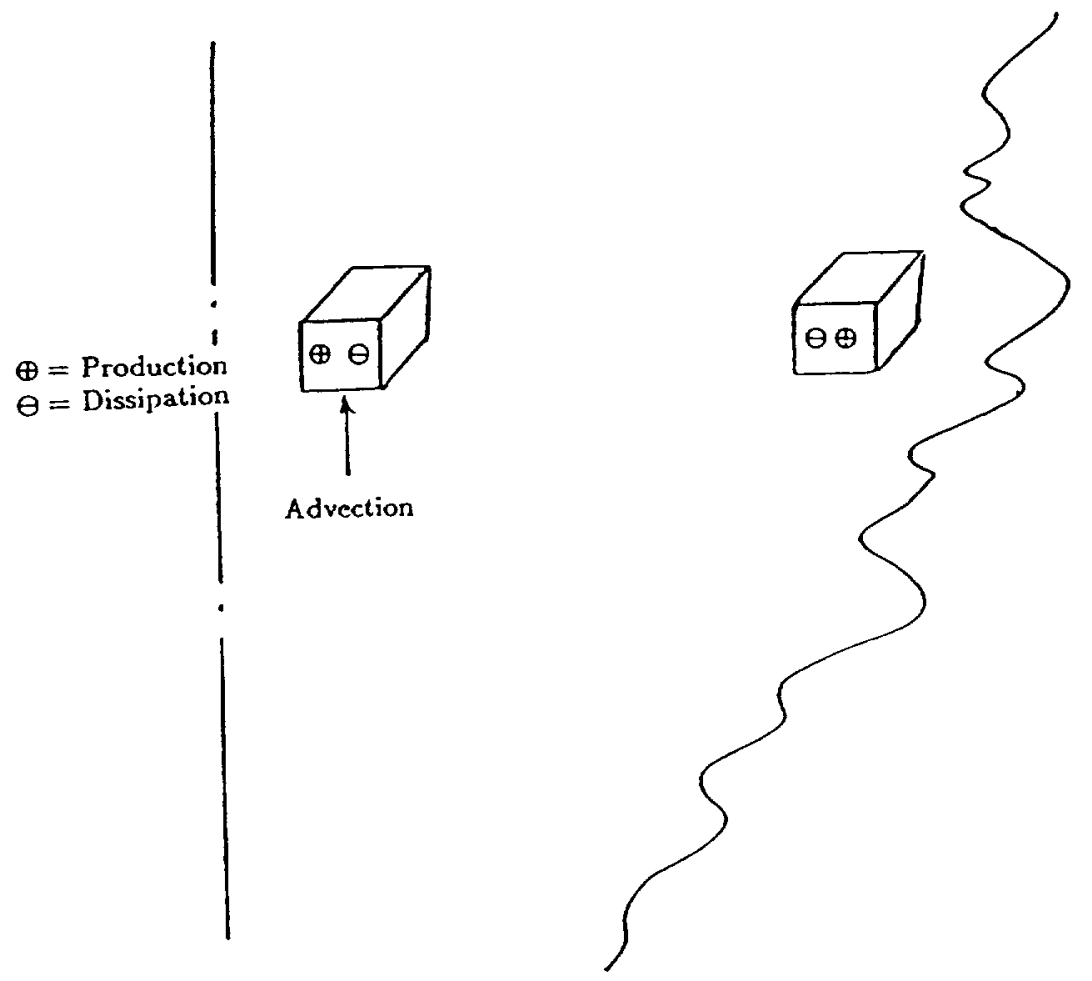

Figure 20b 


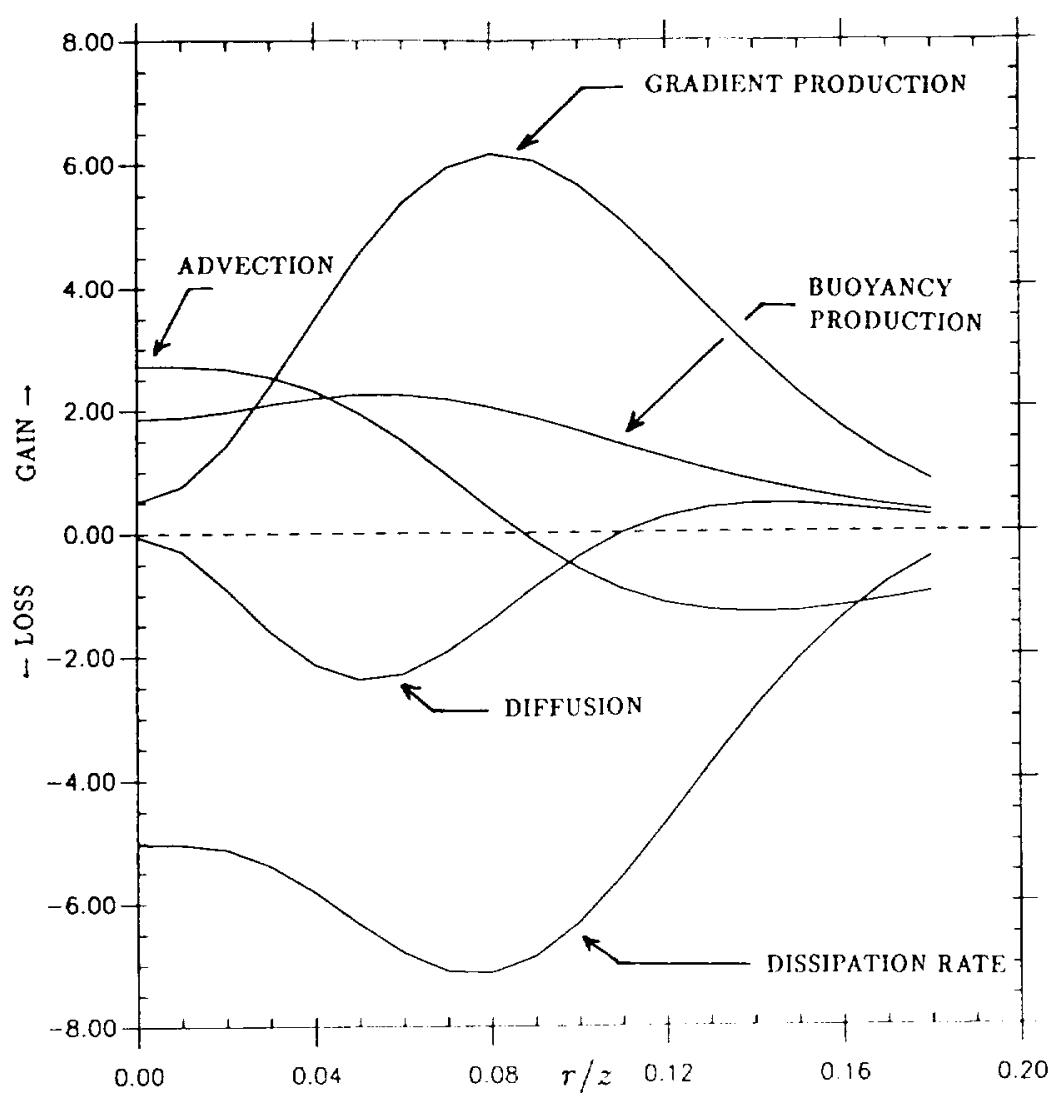

Figure 21a

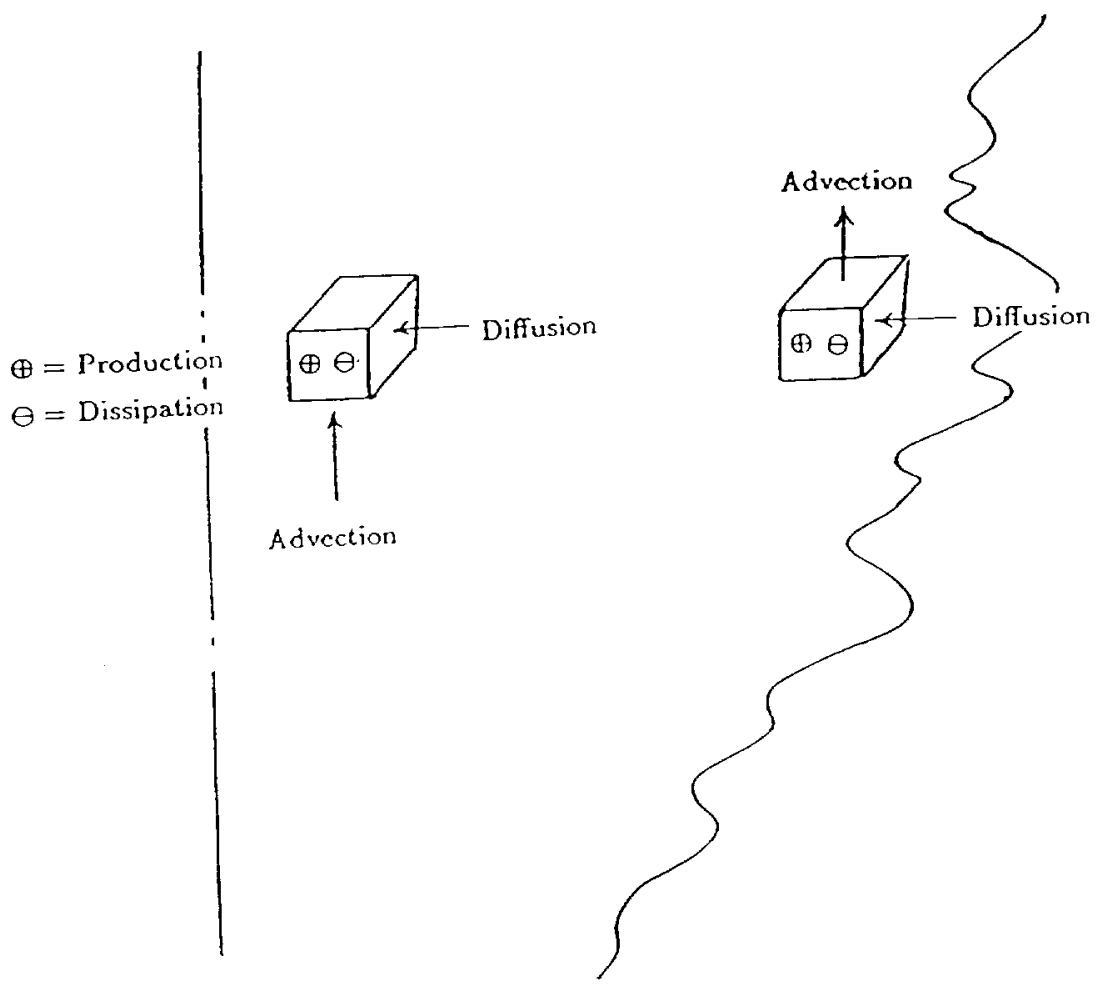

Figure 21b 


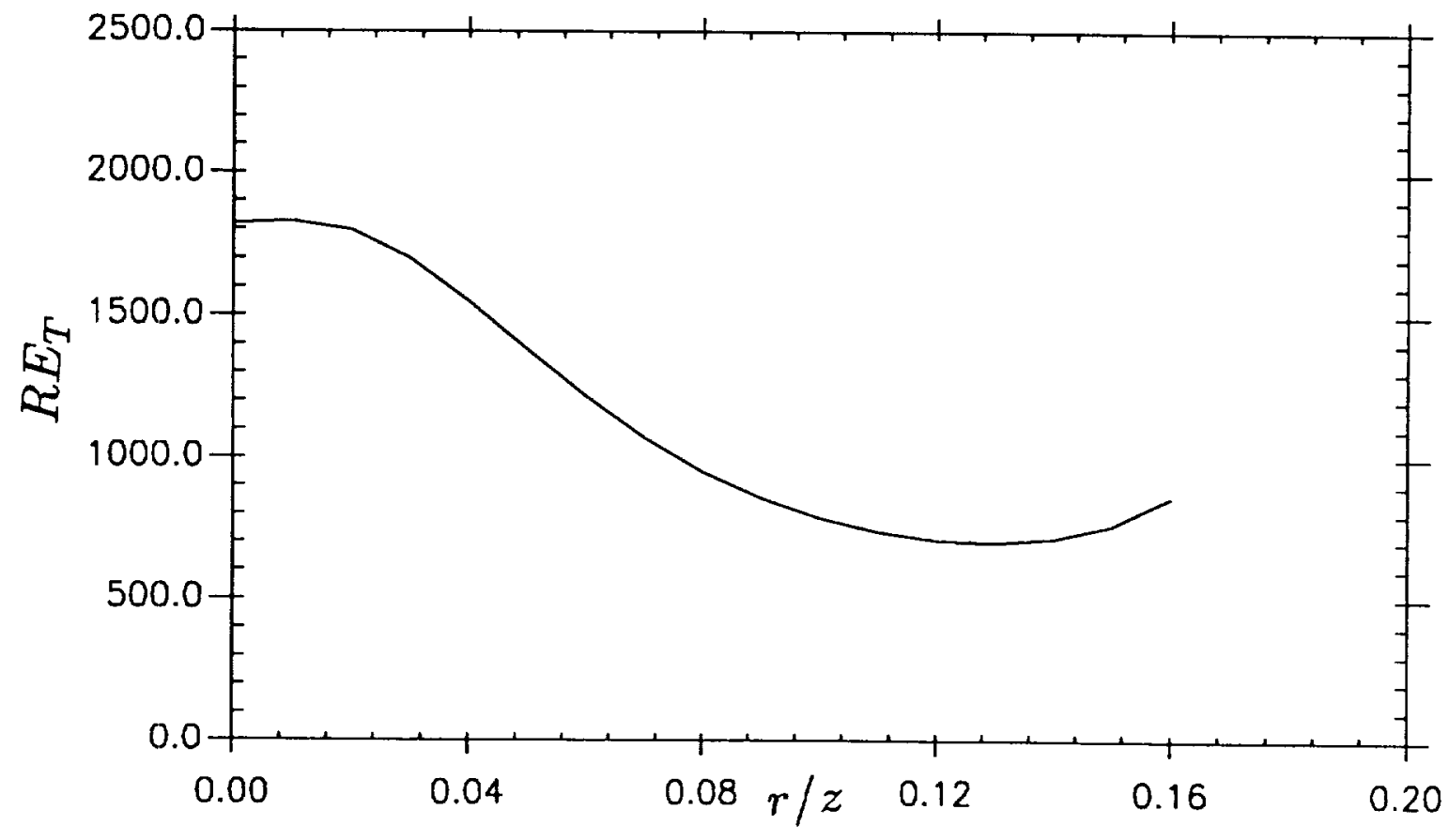

Figure 22 


\section{APPENDIX A}

This appendix gives the recommended polynomial fits to the data and lists the experimental data which is plotted in various figures of the paper. Note that all the data is given in similarity varaibles and can easily be converted into dimensional form by using the appropriate vertical distance and the source buoyancy flux given in Table I. of the main paper.

TABLE A-I - Polynomial Fits to the Experimental Data

$$
\begin{aligned}
& \overline{w^{2}}=\frac{\left(1.1+200 \eta^{2}\right)}{\left(1+38 \eta^{2}\right)^{4}} \\
& \overline{u^{2}}=\frac{\left(0.65+67.35 \eta^{2}-227.26 \eta^{4}\right)}{\left(1+30 \eta^{2}\right)^{4}} \\
& \overline{u w}=\frac{\left(6.5 \eta-104.23 \eta^{3}\right)}{\left(1+24 \eta^{2}\right)^{3}} \\
& \overline{t^{2}}=\frac{\left(12.5+2009.1 \eta^{2}\right)}{\left(1+30 \eta^{2}\right)^{6}} \\
& \overline{w t}=\frac{\left(1.85+680 \eta^{2}\right)}{\left(1+32 \eta^{2}\right)^{6}} \text { or } \overline{w t}=\frac{\left(1.85+580 \eta^{2}\right)}{\left(1+32 \eta^{2}\right)^{6}} \\
& \overline{u t}=\frac{23 \eta}{\left(1+41 \eta^{2}\right)^{3}} \\
& \overline{w^{3}}=\frac{\left(0.38+10.6 \eta^{2}+21593.6 \eta^{4}-371355 \eta^{6}\right)}{\left(1+27 \eta^{2}\right)^{6}} \\
& \overline{u^{3}}=\frac{\left(2.4 \eta+157 \eta^{3}\right)}{\left(1+27 \eta^{2}\right)^{6}} \\
& \overline{u w^{2}}=\frac{\left(-4.6 \eta+2035.3 \eta^{3}-12430.9 \eta^{4}+17881.0 \eta^{5}\right)}{\left(1+27 \eta^{2}\right)^{6}} \\
& \overline{u^{2} w}=\frac{\left(-0.07-26.94 \eta^{2}+2429.21 \eta^{3}-15618.29 \eta^{4}+193866.46 \eta^{6}\right)}{\left(1+27 \eta^{2}\right)^{6}} \\
& \overline{w^{2} t}=\frac{\left(0.75+226 \eta^{2}+11740 \eta^{4}\right)}{\left(1+26 \eta^{2}\right)^{6}} \\
& \overline{u^{2} t}=\frac{\left(-0.338-54.96 \eta^{2}+65229.48 \eta^{4}-561905.62 \eta^{5}+1369428.12 \eta^{6}\right)}{\left(1+27 \eta^{2}\right)^{6}} \\
& \overline{w t^{2}}=\frac{\left(2.9+746.3 \eta^{2}-4309.35 \eta^{6}\right)}{\left(1+23 \eta^{2}\right)^{6}} \\
& \overline{u t^{2}}=\frac{\left(8381.55 \eta^{3}-35932.23 \eta^{4}+98082.8 \eta^{6}\right)}{\left(1+27 \eta^{2}\right)^{6}}
\end{aligned}
$$




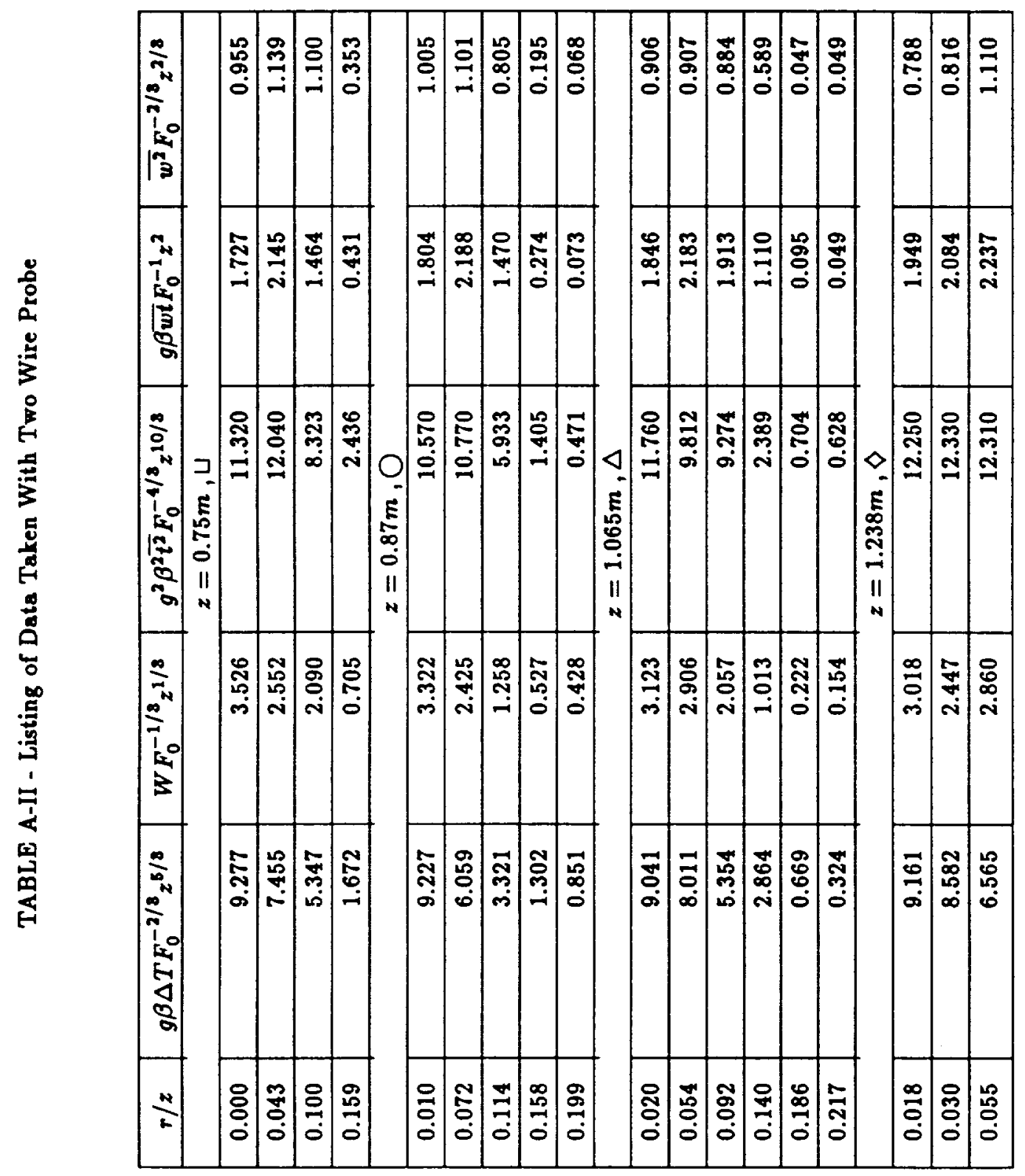




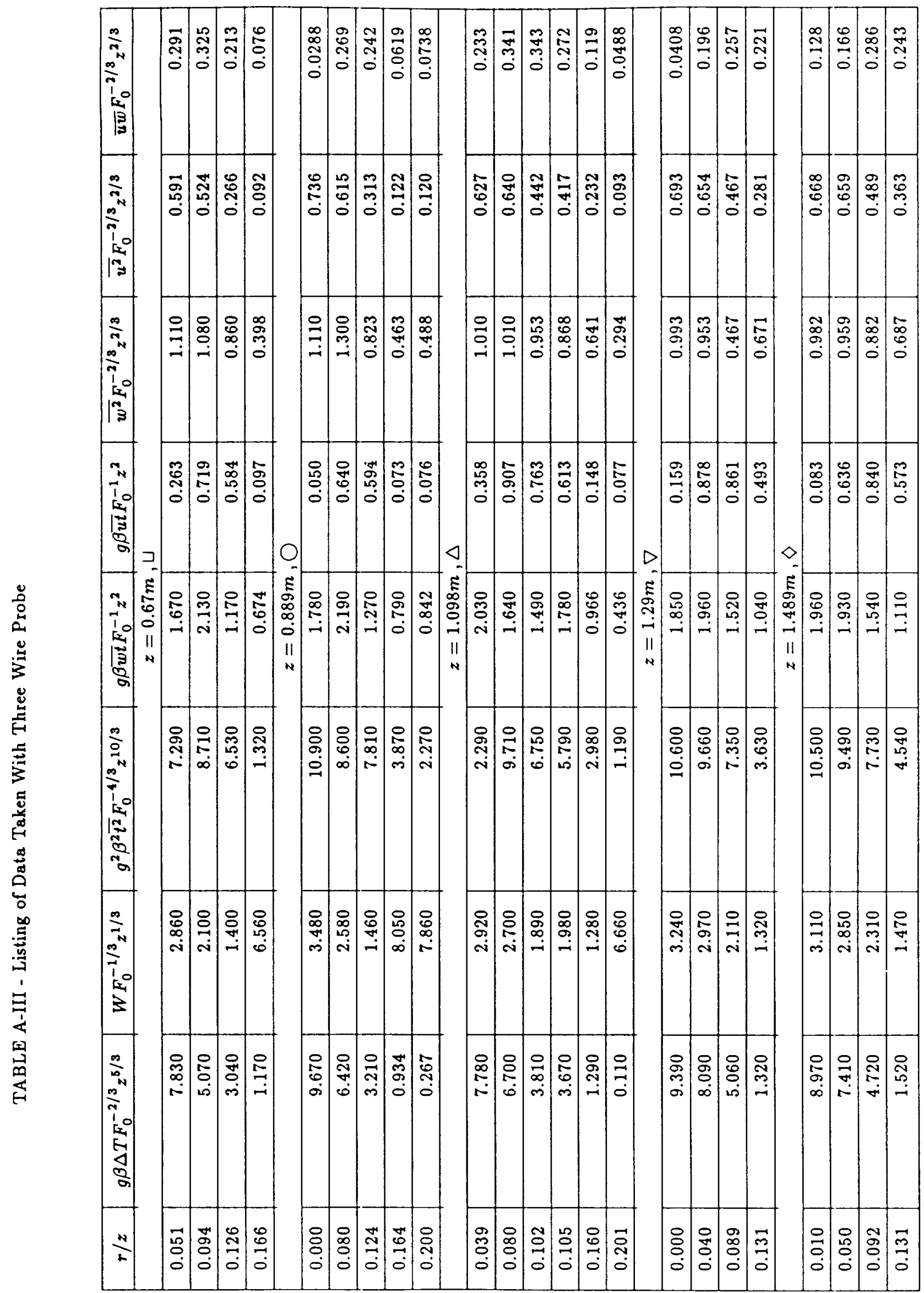


TABLE A-IV - Terms of the Mean Energy Equation

\begin{tabular}{|c|r|r|r|r|r|}
\hline$\eta$ & $\begin{array}{r}\text { Radial } \\
\text { Advection }\end{array}$ & $\begin{array}{c}\text { Vertical } \\
\text { Advection }\end{array}$ & $\begin{array}{c}\text { Radial } \\
\text { Transport }\end{array}$ & $\begin{array}{c}\text { Vertical } \\
\text { Transport }\end{array}$ & Error \\
\hline 0.00 & 0.00 & -53.27 & 44.97 & -3.60 & 11.89 \\
\hline 0.02 & -0.26 & -49.00 & 41.70 & -4.05 & 11.60 \\
\hline 0.04 & -0.72 & -37.86 & 31.00 & -4.64 & 12.22 \\
\hline 0.06 & -0.73 & -23.90 & 18.70 & -4.23 & 10.16 \\
\hline 0.08 & 0.11 & -11.36 & 8.61 & -2.77 & 5.41 \\
\hline 0.10 & 1.59 & -2.78 & 2.10 & -1.09 & 0.18 \\
\hline 0.12 & 3.08 & 1.52 & -1.30 & 0.11 & -3.42 \\
\hline 0.14 & 3.98 & 2.70 & -2.64 & 0.70 & -4.74 \\
\hline 0.16 & 4.05 & 2.30 & -2.87 & 0.84 & -4.33 \\
\hline 0.18 & 3.47 & 1.48 & -2.59 & 0.75 & -3.11 \\
\hline
\end{tabular}

TABLE A-V - Terms of the Mean Momentum Equation

\begin{tabular}{|c|r|r|r|r|r|c|}
\hline$\eta$ & $\begin{array}{c}\text { Radial } \\
\text { Advection }\end{array}$ & $\begin{array}{c}\text { Vertical } \\
\text { Advection }\end{array}$ & $\begin{array}{c}\text { Radial } \\
\text { Transport }\end{array}$ & $\begin{array}{c}\text { Vertical } \\
\text { Transport }\end{array}$ & $\begin{array}{c}\text { Buoyancy } \\
\text { Force }\end{array}$ & Error \\
\hline 0.00 & 0.00 & -3.85 & 12.72 & 0.30 & -9.40 & -0.23 \\
\hline 0.02 & 0.00 & -3.17 & 12.12 & 0.34 & -9.15 & 0.15 \\
\hline 0.04 & 0.00 & -1.42 & 9.76 & 0.37 & -8.43 & 0.28 \\
\hline 0.06 & -0.01 & 0.64 & 6.69 & 0.29 & -7.36 & 0.26 \\
\hline 0.08 & 0.00 & 2.25 & 3.70 & 0.10 & -6.08 & -0.04 \\
\hline 0.10 & 0.03 & 3.00 & 1.31 & -0.11 & -4.76 & -0.54 \\
\hline 0.12 & 0.08 & 2.91 & -0.29 & -0.26 & -3.53 & -1.09 \\
\hline 0.14 & 0.13 & 2.31 & -1.17 & -0.32 & -2.48 & -1.54 \\
\hline 0.16 & 0.15 & 1.56 & -1.53 & -0.32 & -1.65 & -1.78 \\
\hline 0.18 & 0.14 & 0.92 & -1.55 & -0.29 & -1.04 & -1.81 \\
\hline
\end{tabular}

TABLE A-VI - Terms of the Temperature Variance Equation

\begin{tabular}{|c|r|r|r|r|}
\hline$\eta$ & \multicolumn{1}{|c|}{ Advection } & \multicolumn{1}{c|}{ Diffusion } & Production & Dissipation \\
\hline 0.000 & 70.833 & 5.288 & 28.983 & -105.104 \\
\hline 0.020 & 68.197 & 0.238 & 39.897 & -108.331 \\
\hline 0.040 & 59.001 & -7.912 & 61.471 & -112.561 \\
\hline 0.060 & 43.094 & -10.328 & 74.180 & -106.946 \\
\hline 0.080 & 24.952 & -6.232 & 70.351 & -89.072 \\
\hline 0.100 & 10.273 & -0.232 & 55.527 & -65.567 \\
\hline 0.120 & 1.549 & 3.847 & 38.301 & -43.697 \\
\hline 0.140 & -2.129 & 5.160 & 23.882 & -26.913 \\
\hline 0.160 & -2.880 & 4.630 & 13.743 & -15.493 \\
\hline 0.180 & -2.429 & 3.402 & 7.384 & -8.357 \\
\hline
\end{tabular}


TABLE A-VII - Terms of the Turbulence Kinetic Energy

\begin{tabular}{|c|r|r|r|r|r|}
\hline$\eta$ & Advection & Diffusion & $\begin{array}{c}\text { Gradient } \\
\text { Production }\end{array}$ & $\begin{array}{c}\text { Buoyancy } \\
\text { Production }\end{array}$ & Dissipation \\
\hline 0.0110 & 2.720 & -0.051 & 0.511 & 1.850 & -5.030 \\
\hline 0.020 & 2.656 & -0.912 & 1.413 & 1.966 & -5.123 \\
\hline 0.040 & 2.299 & -2.141 & 3.481 & 2.177 & -5.816 \\
\hline 0.060 & 1.475 & -2.289 & 5.381 & 2.234 & -6.801 \\
\hline 0.080 & 0.380 & -1.426 & 6.147 & 2.028 & -7.129 \\
\hline 0.100 & -0.580 & -0.392 & 5.652 & 1.635 & -6.315 \\
\hline 0.120 & -1.147 & 0.250 & 4.373 & 1.198 & -4.675 \\
\hline 0.140 & -1.312 & 0.448 & 2.910 & 0.818 & -2.863 \\
\hline 0.160 & -1.211 & 0.387 & 1.675 & 0.531 & -1.382 \\
\hline 0.180 & -0.992 & 0.246 & 0.823 & 0.334 & -0.411 \\
\hline
\end{tabular}




\section{APPENDIX B}

The purpose of this appendix is to show the sensitivity of the boundary conditions on the growth of a laboratory plume. Our experience indicates that the differences in various experiments are mostly due to the differences in their boundary conditions rather than differences in their instrumentation. This sensitivity to the boundary conditions is studied in the current work by placing screens of different sizes around the plume source. These screens are employed in such experiments in an attempt to keep the cross-drafts and other disturbances away from the flow. This is desirable because the velocities encountered in laboratory plumes are small $(\sim 1 \mathrm{~m} / \mathrm{s})$ and because of this the flow can easily be effected by any small currents or disturbances present in the facility. These screens also help to keep the plume from meandering. It is always presumed that these screens do not influence the laboratory flow because these are usually placed well away from the edges of the flow and the measurements are taken at heights far above the source. In this appendix first an order of magnitude analysis of the mean momentum equation is carried out in order to show that screens influence the flow by modifying the entrainment flow. Afterwards mean flow and turbulence measurements are presented to support the analysis.

The appropriate scaling for a boundary free turbulent shear flow is $\frac{\theta}{\partial z} \approx \frac{1}{L}$ and $\frac{\theta}{\partial r} \approx \frac{1}{\delta}$ where $\delta$ is the width of the flow and $\frac{\delta}{L}<<1$. By scaling the mean velocity $W$ by $W$, and all the turbulent velocities by $w_{s}$ the order of various terms in vertical and radial momentum equations for the round plume are

$$
\begin{aligned}
& U \frac{\partial W}{\partial r}+W \frac{\partial W}{\partial z}=-\frac{1}{\rho} \frac{\partial}{\partial z} P-\frac{1}{r} \frac{\partial}{\partial r}(r \overline{u w})-\frac{\partial}{\partial z} \overline{w^{2}}+g \beta \Delta T \\
& 1 \quad 1 \quad ? \quad \frac{w_{s}^{2}}{W_{s}} \frac{L}{\delta} \quad \frac{w_{s}^{2}}{W_{s}} \quad g \beta \frac{T_{s} L}{W_{s}^{2}}
\end{aligned}
$$




$$
\begin{aligned}
& U \frac{\partial U}{\partial r}+W \frac{\partial U}{\partial z}=-\frac{1}{\rho} \frac{\partial}{\partial r} P-\frac{1}{r} \frac{\partial}{\partial r}\left(r \overline{u^{2}}\right)-\frac{\partial}{\partial z} \overline{u w}-\frac{\overline{v^{2}}}{r} \\
& \frac{\delta}{L} \quad \frac{\delta}{L} \quad ? \quad \frac{L}{\delta} \frac{w_{s}^{2}}{W_{s}^{2}} \quad \frac{w_{s}^{2}}{W_{s}^{2}} \quad \frac{w_{s}^{2}}{W_{s}^{2}} \frac{L}{\delta}
\end{aligned}
$$

In the above, $U$ is scaled as $W_{s} \delta / L$ which is obtained from the continuity equation.

Keeping only the leading terms, the radial momentum equation can be integrated from infinity to a given radius to obtain

$$
P_{\infty}-P \approx \frac{\rho\left(\overline{u^{2}}+\overline{v^{2}}\right)}{2} \approx \rho \overline{u^{2}}
$$

Therefore, the entrainment, which is driven by the above pressure difference, is of second order. Since $\rho \overline{u^{2}}$ is already a second order quantity, the above relation implies that a third order term can influence the entrainment and hence the plume growth.

Now consider a screen placed around the plume source as shown in figure B1. The effect of the screen is to reduce the pressure drop driving the entrainment, i.e.

$$
P_{\infty}-P \approx \rho \overline{u^{2}}+\Delta P_{\text {screen }}
$$

If the screen is modeled by a simple pressure coefficient, then

$$
P_{\infty}-P \approx \rho \overline{u^{2}}+\frac{1}{2} K \rho U_{s}^{2}
$$

where $K$ is the screen constant and $U_{s}$ is the entrainment velocity at the location where the screen is placed. From (B.7) we see that the screen will leave the entrainment field unchanged only if

$$
\frac{1}{2} K \rho U_{s}^{2}<<\overline{u^{2}}
$$

For round geometry this can always be satisfied by placing the screens far enough away since $U \rightarrow 1 / r$ as $r \rightarrow \infty$. (Note that the measurements to be presented next do not correspond to such a case.) 
To support the above analysis mean buoyancy profiles were measured, using thermocouples, by placing three different screens around the plume source (see figure B2). Figure B3 shows the mean buoyancy profiles corresponding to these screens along with the profile measured without using any screen. We note that the effect of the screens is to make the buoyancy profiles narrower and higher. This is because the screens reduce the entrainment of the surrounding cooler air into the main flow as was suggested by the above analysis.

More detailed hot wire measurements were carried out for screen $\mathrm{C}$ in order to document the screen effects on mean as well as turbulence quantities. For this purpose the two wire probe, described irı section 3.2, was used. The results are presented in figures B4-B8. We note that the data collapse is excellent (and even better than the measurements presented in the main paper). The measurements satisfy the integral energy constraint given by equation (12) (these results are given in Shabbir 1987). This shows that the collapse of experimental data into similarity variables and conservation of buoyancy (or any other relevant integral constraint) does not necessarily mean that the experiment represents the flow which one set out to measure. One must probe the facility carefully to make sure that no external influences are present.

The above analysis can be extended to cases where the boundary conditions of the experiment involve weak co-flowing streams etc. since these also modify the entrainment flow. This is discussed in detail in a review paper by George (1990). 


\section{FIGURE CAPTIONS FOR APPENDIX B}

Figure B1. Sketch of the radial distribution of pressure near the plume source with and without a screen around it's source.

Figure B2. Sketch showing the placement of screens around the plume source and the size of the three screens used to study their effect on plume growth.

Figure B3. The mean buoyancy profiles measured, using thermocouples, with the three screens shown in figure B2 placed around the plume source and the buoyancy profile measured without using such a screen.

Figure B4. Mean buoyancy profile measured with a hot-wire probe with the screen $C$ placed around the plume source. $\quad, z / D=15 ; \quad \mathbf{A}, \mathrm{z} / \mathrm{D}=17 ; \mathbf{0}, \mathrm{z} / \mathrm{D}=19 ; \quad \boldsymbol{\mathrm { v }}, \mathrm{z} / \mathrm{D}=21$.

Figure B5. Mean velocity profile measured with the screen $\mathrm{C}$ placed around the source. For symbols see caption for figure B4.

Figure B6. Intensity of temperature fluctuations measured with the screen $C$ placed around the plume source. For symbols see caption for figure B4.

Figure B7. Intensity of velocity fluctuations with screen $\mathrm{C}$ placed around the plume source. For symbols see caption for figure B4.

Figure B8. Correlation coefficient between the vertical velocity fluctuations and the temperature fluctuations measured with the screen $C$ placed around the plume source. For symbols see caption for figure B5. 

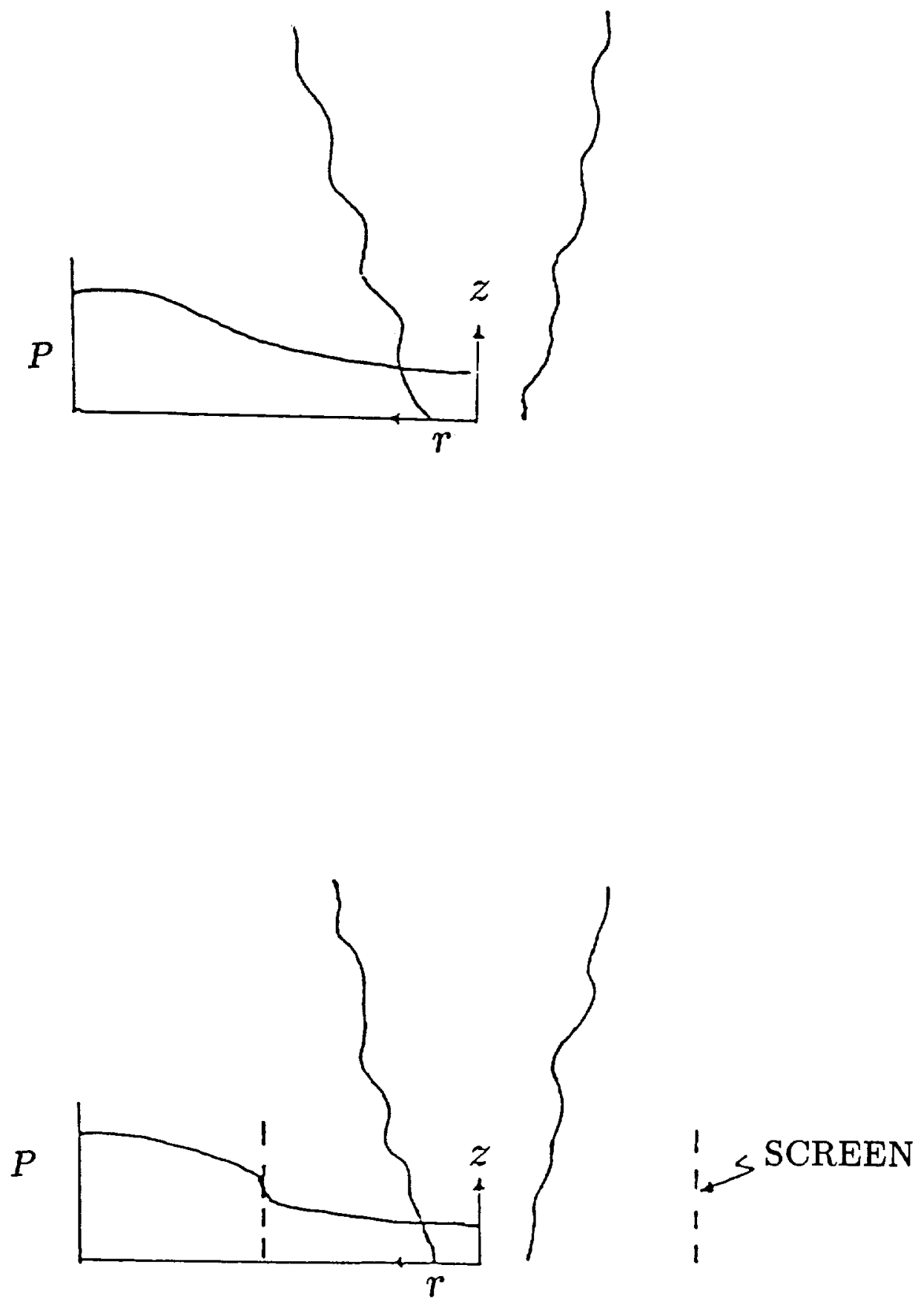

Figure B1 

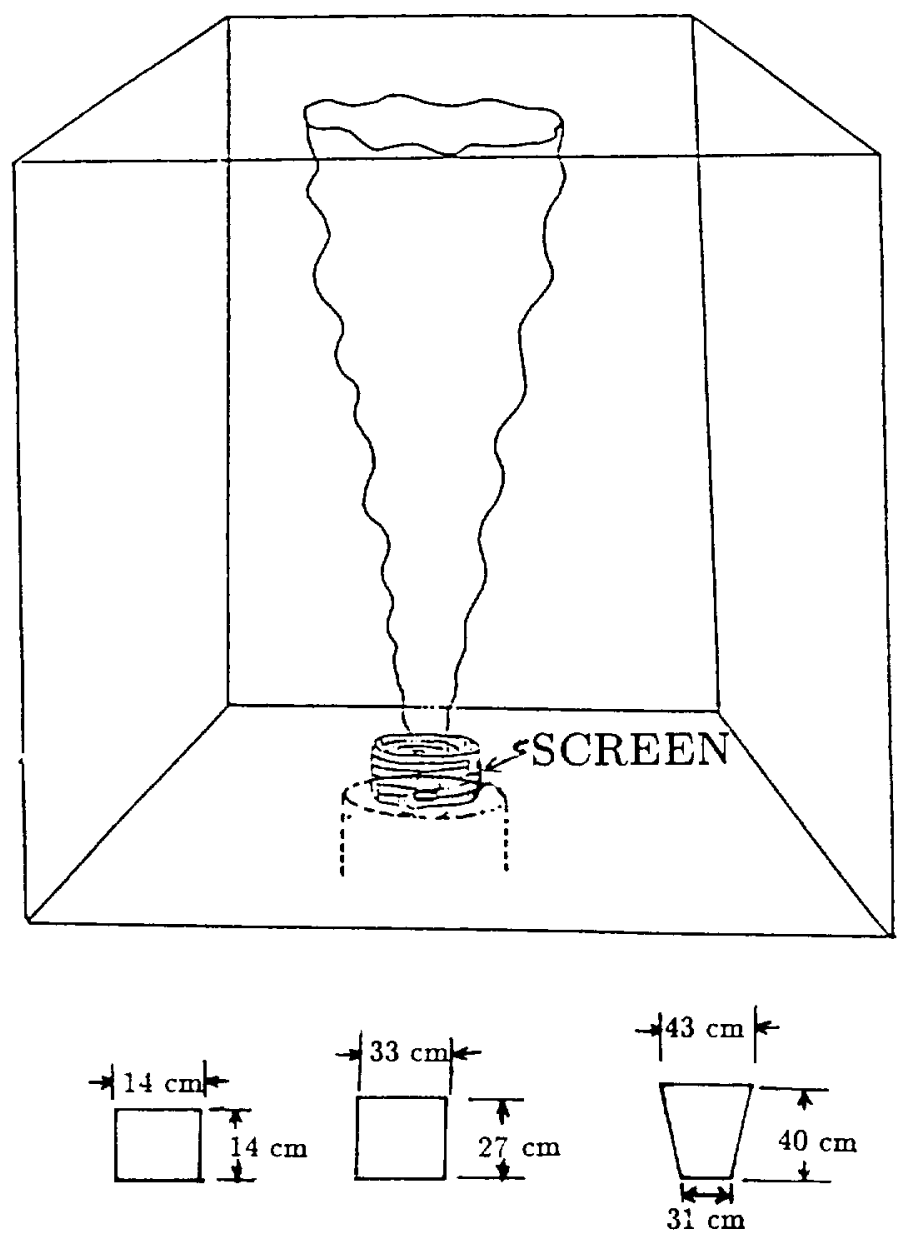

A

B

C

Figure B2 


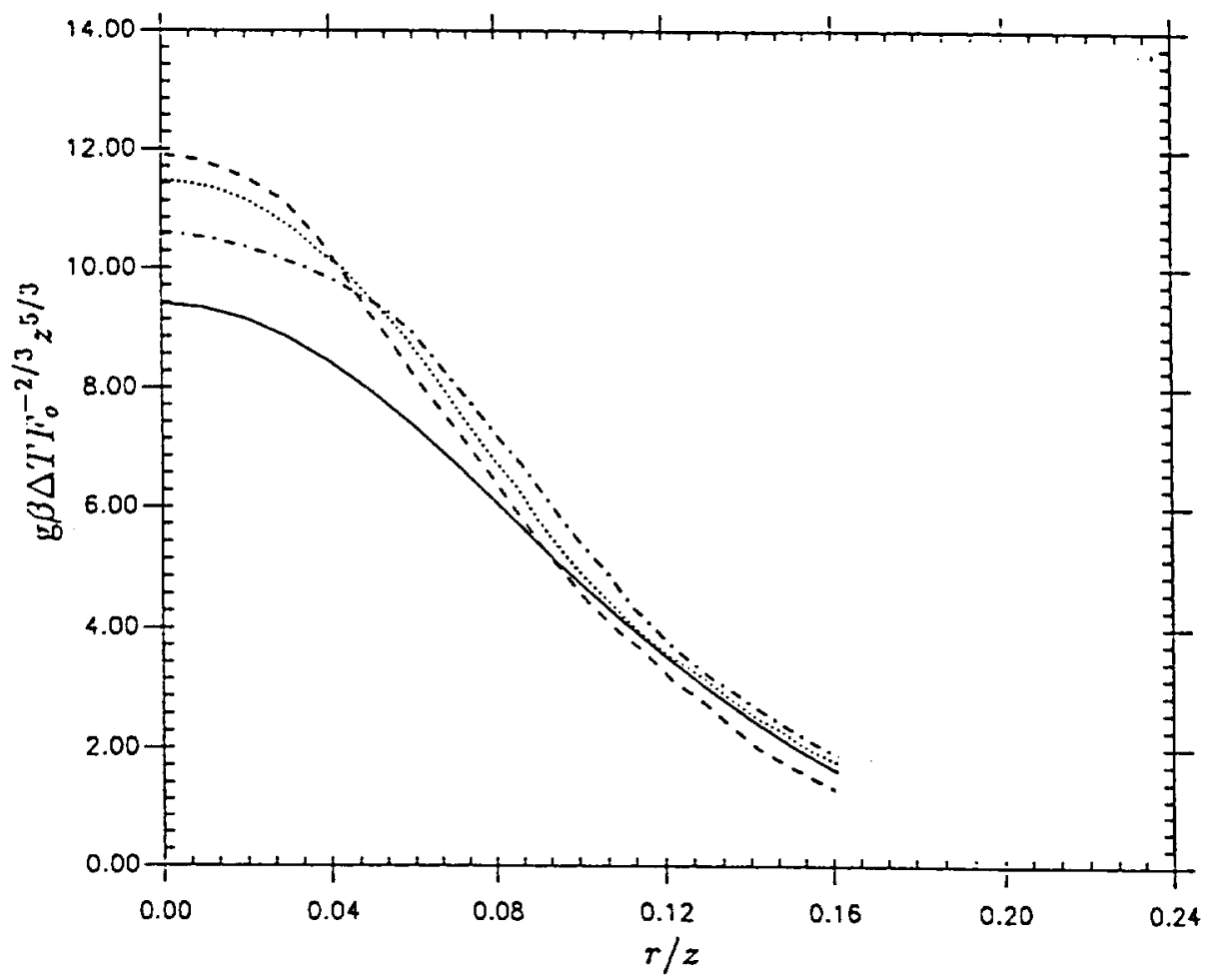

Figure B3

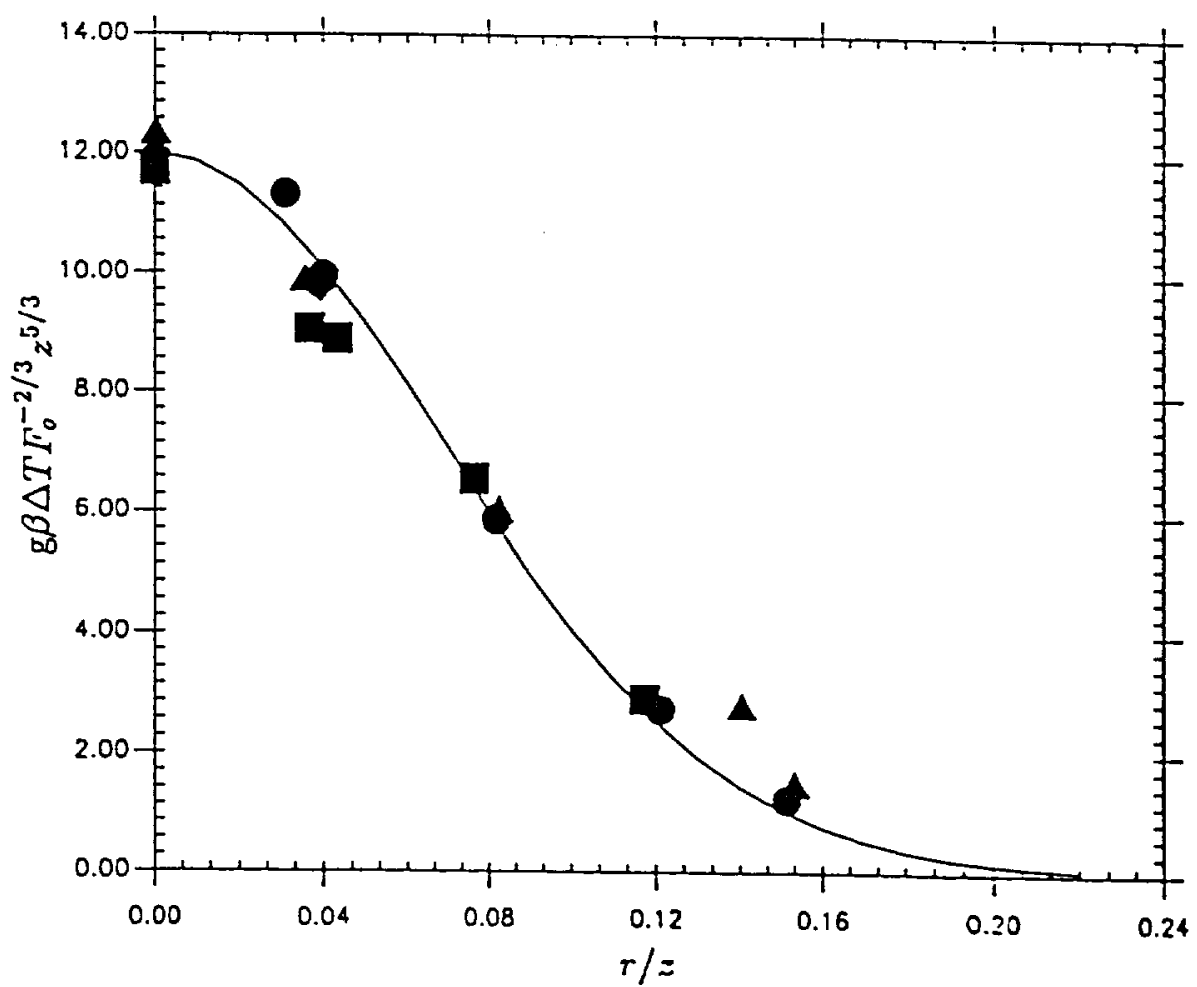

Figure B4 


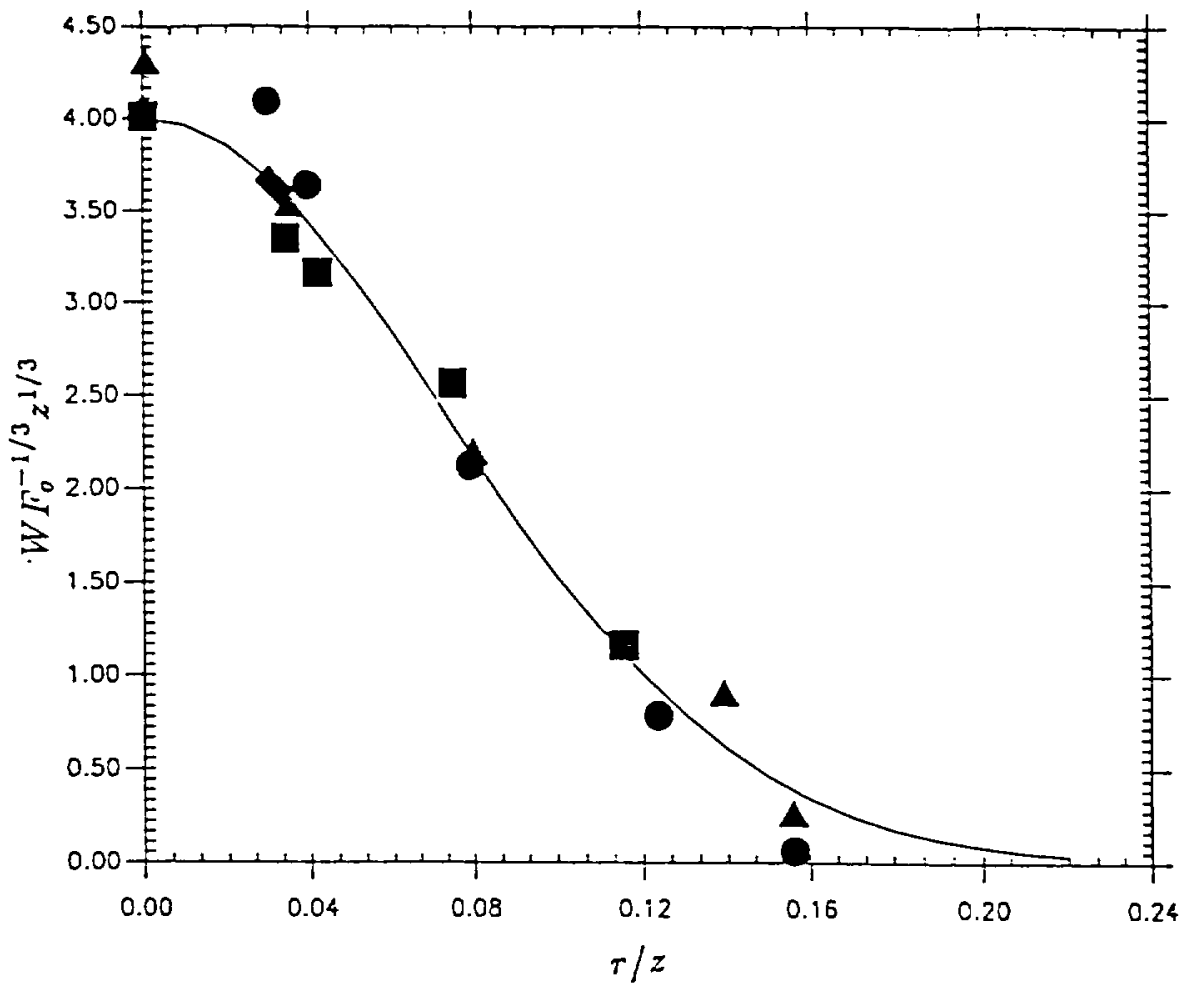

Figure B5

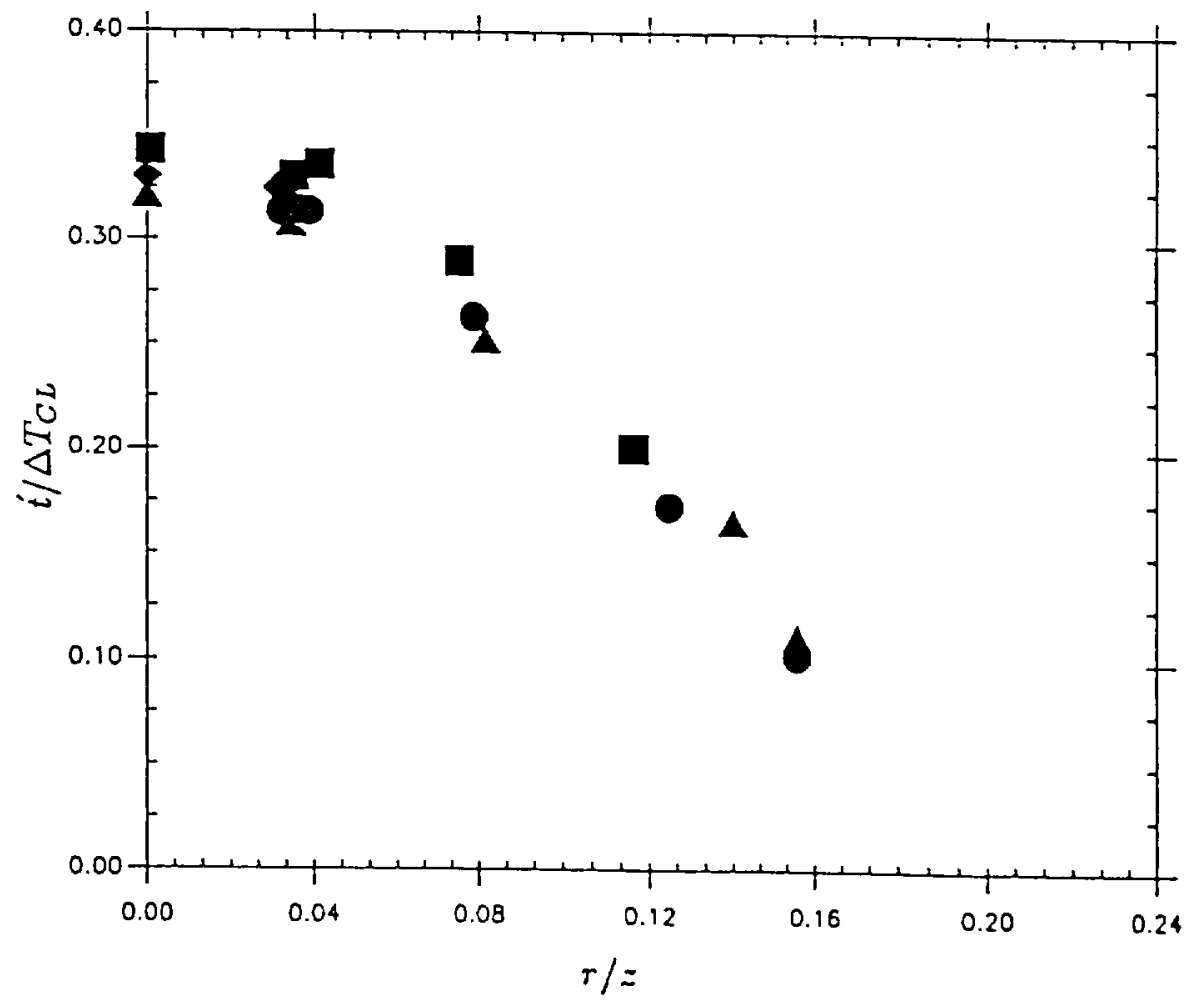

Figure B6 


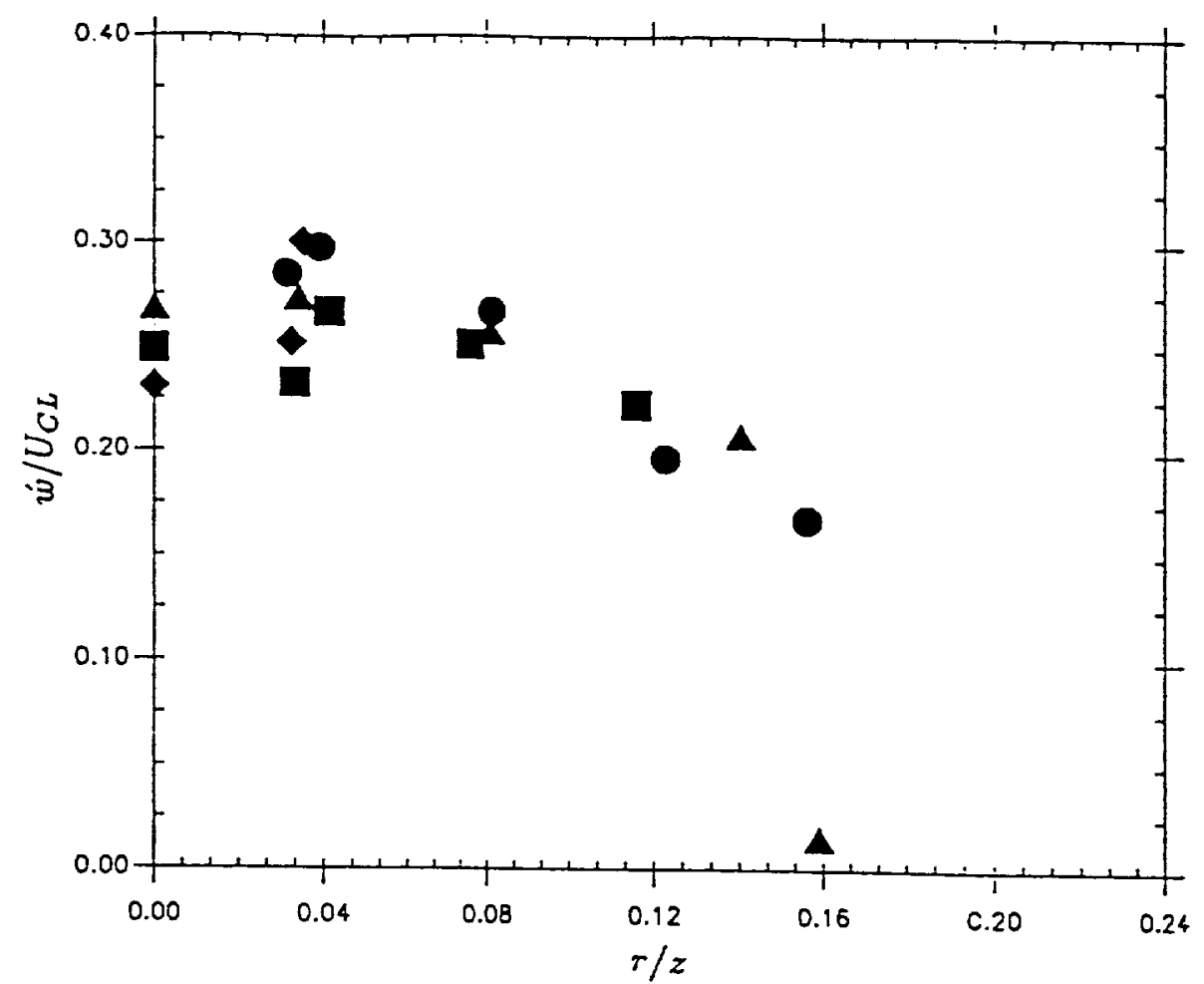

Figure B7

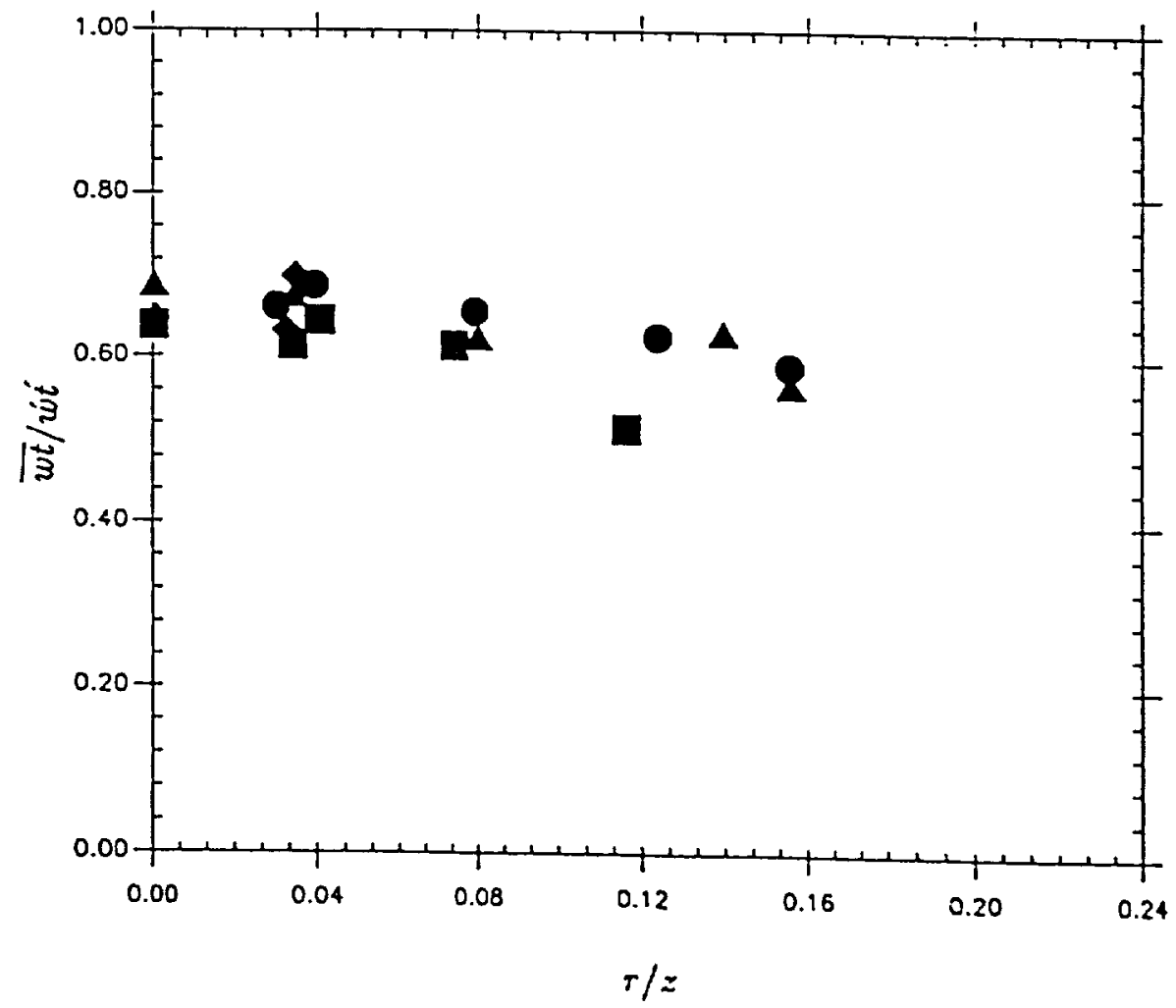

Figure B8 

